\title{
The epithelial-to-mesenchymal transition activator ZEB1 initiates a prometastatic competing endogenous RNA network
}

\author{
Xiaochao Tan, ${ }^{1}$ Priyam Banerjee, ${ }^{1}$ Xin Liu, ${ }^{1}$ Jiang Yu, ${ }^{1}$ Don L. Gibbons, ${ }^{1,2}$ Ping Wu, ${ }^{3,4}$ Kenneth L. Scott, ${ }^{3,4}$ Lixia Diao, ${ }^{5}$ \\ Xiaofeng Zheng, ${ }^{5}$ Jing Wang, ${ }^{5}$ Ali Jalali, ${ }^{6}$ Milind Suraokar, ${ }^{7}$ Junya Fujimoto, ${ }^{7}$ Carmen Behrens, ${ }^{1,7}$ Xiuping Liu, ${ }^{8}$ Chang-gong Liu, ${ }^{8}$ \\ Chad J. Creighton, ${ }^{4,5}$ Ignacio I. Wistuba, ${ }^{1,7}$ and Jonathan M. Kurie ${ }^{1}$ \\ 'Department of Thoracic/Head and Neck Medical Oncology and '2Department of Molecular Oncology, The University of Texas MD Anderson Cancer Center, Houston, Texas, USA. ${ }^{3}$ Department of Molecular and \\ Human Cenetics and ${ }^{4}$ Department of Medicine and Dan L. Duncan Cancer Center, Baylor College of Medicine, Texas, USA. ${ }^{5}$ Department of Bioinformatics and Computational Biology, The University of Texas \\ MD Anderson Cancer Center, Houston, Texas, USA. ${ }^{6}$ Department of Neurosurgery, Baylor College of Medicine, Houston, Texas, USA. 'Department of Translational Molecular Pathology, Division of Pathology \\ and Laboratory Medicine, and ${ }^{8}$ Department of Experimental Therapeutics, The University of Texas MD Anderson Cancer Center, Houston, Texas, USA.
}

\begin{abstract}
Epithelial tumor cells undergo epithelial-to-mesenchymal transition (EMT) to gain metastatic activity. Competing endogenous RNAs (ceRNAs) have binding sites for a common set of microRNAs (miRs) and regulate each other's expression by sponging miRs. Here, we address whether ceRNAs govern metastasis driven by the EMT-activating transcription factor ZEB1. High miR-181b levels were correlated with an improved prognosis in human lung adenocarcinomas, and metastatic tumor cell lines derived from a murine lung adenocarcinoma model in which metastasis is ZEB1-driven were enriched in miR-181b targets. ZEB1 relieved a strong basal repression of $\alpha_{1}$ integrin (ITCA1) mRNA, which in turn upregulated adenylyl cyclase 9 mRNA (ADCY9) by sponging miR181b. Ectopic expression of the ITCA1 3'-untranslated region reversed miR-181b-mediated metastasis suppression and increased the levels of adenylyl cyclase 9 protein (AC9), which promoted tumor cell migration and metastasis. In human lung adenocarcinomas, ITCA1 and ADCY9 levels were positively correlated, and an AC9-activated transcriptomic signature had poor-prognostic value. Thus, ZEB1 initiates a miR-181b-regulated ceRNA network to drive metastasis.
\end{abstract}

\section{Introduction}

Metastasis is the primary cause of cancer-related death (1) and is driven by tumor cells that are uniquely capable of switching reversibly between epithelial and mesenchymal states in response to extracellular cues (2). During epithelial-to-mesenchymal transition (EMT), tumor cells switch their axis of polarity from apicalbasal to front-rear, detach from neighboring cells, and become more motile and invasive and more resistant to cytotoxic chemotherapy (3-6). EMT is activated by several transcription factor families (ZEB, SNAIL, TWIST, FOXO, and others) (7). In a pancreatic cancer model driven by PDX1-Cre-mediated activation of mutant Kras and p53 alleles, multiple EMT-activating transcription factors (EMT-TFs) are upregulated, and metastasis is ablated by inactivation of ZEB1 but not SNAIL or TWIST $(8,9)$, suggesting variable prometastatic activities. In breast and lung adenocarcinomas, high ZEB1 expression levels confer tumor-initiating and metastatic activities and are correlated with a poor prognosis (10). ZEB1 downregulates the expression of epithelial polarity genes and microRNAs (miRs; e.g., miR-34a, miR-148a, and miR-200 family members) that promote epithelial differentiation and inhibit tumor

Authorship note: KLS is deceased.

Conflict of interest: The authors have declared that no conflict of interest exists.

Submitted: September 1, 2017; Accepted: January 9, 2018.

Reference information: / Clin Invest. 2018;128(4):1267-1282.

https://doi.org/10.1172/JCI97225. initiation and metastasis by regulating actin cytoskeletal remodeling, Golgi compaction, and polarized vesicle trafficking $(4,11,12)$. Thus, miRs are critical downstream effectors of EMT-TFs.

Competing endogenous RNAs (ceRNAs) have binding sites for a common set of miRs and regulate each other's expression by functioning as miR sponges. For example, the phosphatase and tensin homolog (PTEN) pseudogene PTENP1 was found to compete with PTEN for binding to miR-19b and miR-20a; hence, in tumor cells with a WT PTEN locus, loss of PTENP1 leads to miRmediated depletion of PTEN (13). Other noncoding RNA species that can function as ceRNAs include a long noncoding RNA activated by TGF- $\beta$, IncRNA-ATB, which competes with ZEB1 and ZEB2 for binding to miR-200 to drive the invasion-metastasis cascade in hepatocellular carcinoma (14). High levels of circular RNAs such as circRNA_100290 and circRNA_CCDC66 can promote oral cancer and colon cancer, respectively, by functioning as miR sponges $(15,16)$. In addition, the MYCN proto-oncogene drives neuroblastoma progression by sponging let-7 (17). Thus, noncoding RNAs participate in ceRNA networks to drive tumorigenesis, but whether ceRNAs are upregulated during EMT and mediate metastasis driven by EMT-TFs remains unclear.

To test the hypothesis that EMT-TFs initiate ceRNA networks to promote metastasis, we used clinically annotated human lung adenocarcinomas and lung adenocarcinoma cell lines that were derived from mice that express $\mathrm{K}-\mathrm{ras}^{\mathrm{G} 12 \mathrm{D}}$ and $\mathrm{p} 53^{\mathrm{R} 172 \mathrm{H}}$ (hereafter termed KP cells) and differ in metastatic activity (18). Highly 

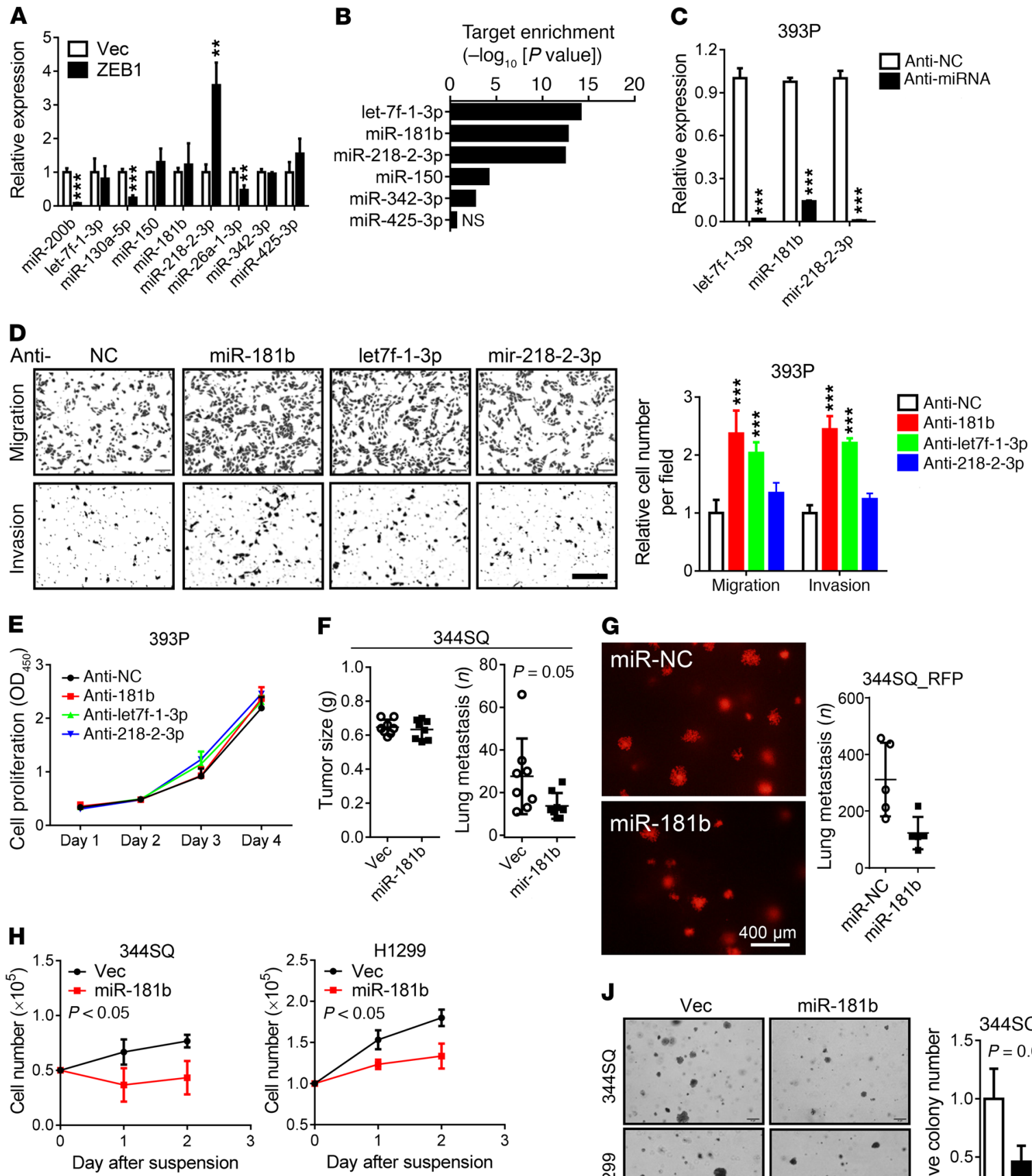

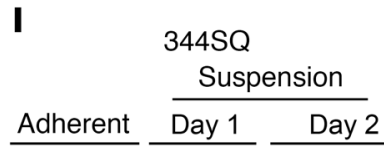

G
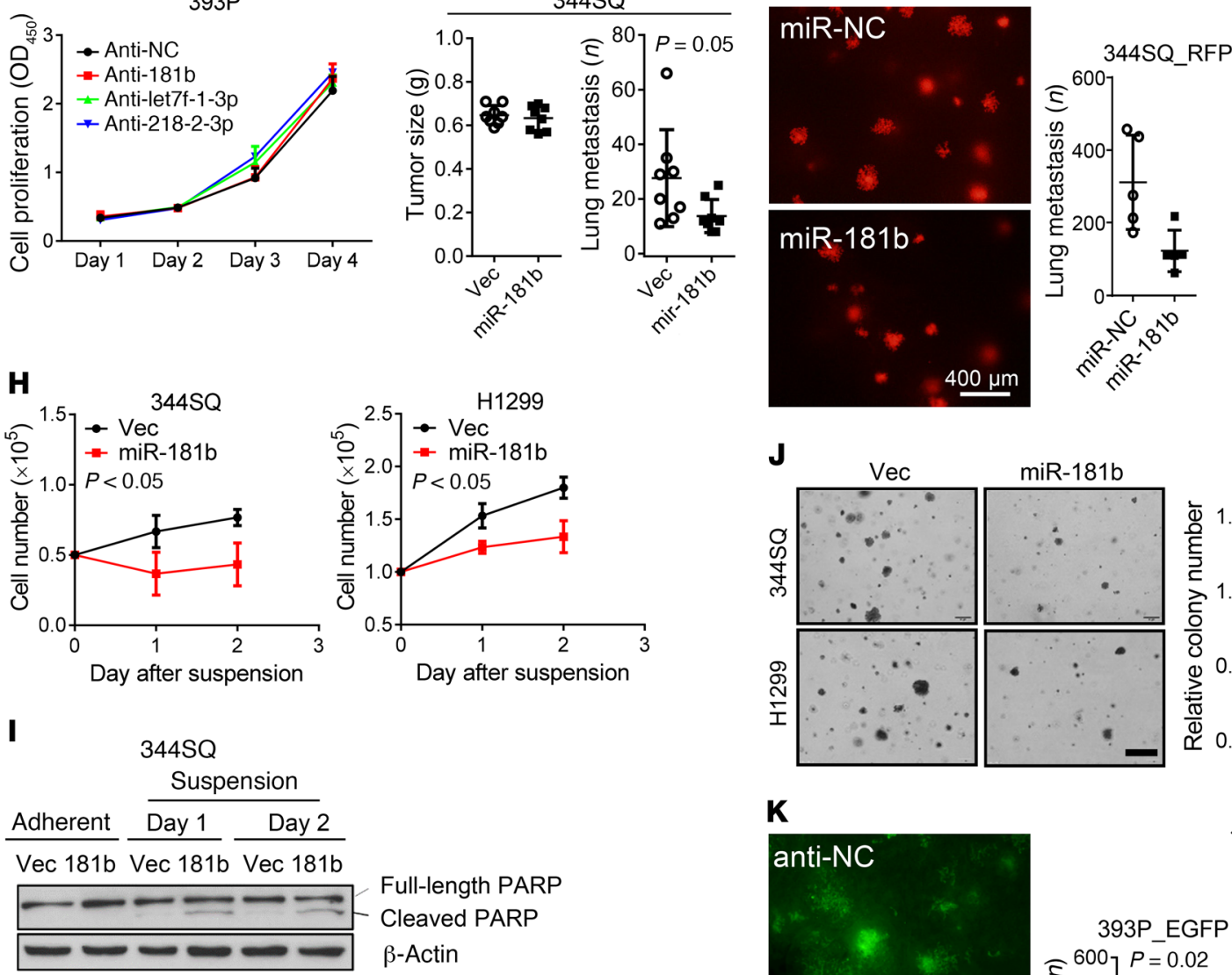

Full-length PARP

Cleaved PARP

$\beta$-Actin

H1299

$\frac{\text { Adherent }}{\frac{\text { Suspension }}{\text { Day } 1}} \frac{\text { Day } 2}{\text { Vec 181b }}$

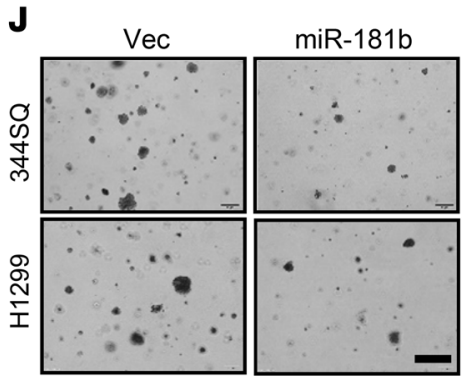

K

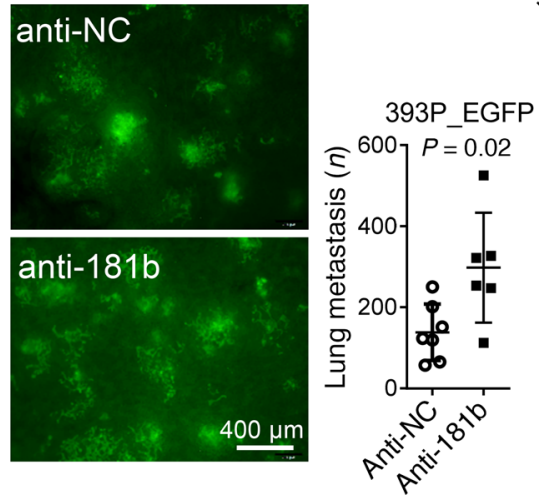


Figure 1. miRs not repressed by ZEB1 have metastasis suppressor activity. (A) Quantitative reverse transcription PCR (qPCR) analysis of miRs in 393P_ZEB1 cells (ZEB1) and 393P_vector cells (Vec). Results expressed relative to 393P_vector cells. miR-200b included as positive control. (B) Enrichment of ZEB1-upregulated genes in miR targets as assessed by a hypergeometric test. (C) qPCR assays quantify antagomir-mediated depletion of miRs in 393P cells. Results expressed relative to control antagomirs (anti-NC). (D) Boyden chamber assays on antagomir- and control-transfected cells. Migratory and invasive cells were photographed (images) and counted (bar graph). Scale bar: $200 \mu \mathrm{m}$. (E) Relative densities of transfectants on plastic. (F) Primary tumor weights (left) and numbers of lung metastases (right) in mice injected subcutaneously with 344SQ cells that express ectopic miR-181b or vector (Vec). (G) Fluorescence microscopic images of lung metastases in mice injected by tail vein with red fluorescent protein-labeled (RFP-labeled) 344SQ cells transfected with miR-181b or control mimics. Scatter plot: numbers of lung metastases per mouse (dots). (H) Numbers of viable $344 \mathrm{SQ}$ and $\mathrm{H} 1299$ transfectants on low-adhesion plates. $n=4$. (I) Western analysis detects cleaved PARP as a marker of apoptosis in transfectants on adhesive or low-adhesion (suspension) plates. $\beta$-Actin is loading control. (J) Soft agar colony formation by miR-181b and vector control (Vec) transfectants. Colonies were photographed (images) and counted (bar graphs). Scale bar: $100 \mu \mathrm{m}$. Results expressed relative to vector controls. (K) Fluorescence microscopic images of lung metastases in mice injected by tail vein with green fluorescent protein-labeled (GFP-labeled) 393P cells transfected with anti-181b or antiNC. Scatter plot: numbers of lung metastases per mouse (dots). Values are mean \pm SD. $n=3$, unless otherwise indicated. $P$ values, 2 -tailed Student's $t$ test and Dunnett's test for 2-group comparisons and comparisons of more than 2 groups, respectively. Results were replicated ( $n \geq 2$ experiments). ${ }^{*} P<0.01$ and ${ }^{* *} P<0.001$.

metastatic KP cell lines undergo a reversible EMT in response to extracellular cues and demonstrate migratory and invasive activities that are driven by high ZEB1 expression levels, whereas poorly metastatic KP cells do not exhibit such differentiation plasticity and remain in an epithelial state (18). The findings presented here support a model in which ZEB1 increases metastasis through a miR-181b-regulated ceRNA network involving $\alpha_{1}$ integrin (ITGA1) and adenylyl cyclase 9 (ADCY9).

\section{Results}

ZEB1 upregulates ITGA1 to inactivate the metastasis suppressor miR$181 b$. To identify miRs that govern an EMT-regulated, prometastatic ceRNA network, we first screened for miRs that are correlated with an improved prognosis in lung cancer, are not silenced during EMT, have targets that are enriched in the ZEB1 transcriptome, and function as suppressors of ZEB1-driven metastasis. By mining expression profiles of over 900 miRs in clinically annotated human lung cancers $(n=246)$ and a poorly metastatic KP cell line (393P) that undergoes EMT and gains invasive and metastatic capabilities after ectopic ZEB1 expression (11), we identified 8 miRs that were correlated with an improved prognosis and were not suppressed by ectopic ZEB1 expression (Supplemental Figure 1A; supplemental material available online with this article; https://oi.org/10.1172/JCI97225DS1). To validate these findings, we performed quantitative reverse transcription PCR (qPCR) analysis on KP cell lines that have been classified as mesenchymal or epithelial (11) and on 393P_ZEB1 cells and 393P_vector cells, which confirmed that 6 of the 8 miRs were not silenced during EMT (Figure 1A and Supplemental Figure 1B). To determine whether the ZEB1 transcriptome is enriched in predicted targets of the 6 miRs, we used a prediction algorithm (www.targetscan. org) to query RNA-Seq data from 393P_ZEB1 cells and 393P_ vector cells and found that the genes upregulated at least 2-fold by ectopic ZEB1 expression were enriched in predicted targets of 5 miRs (Figure 1B).

Next, we performed gain- and loss-of-function studies on 3 miRs that demonstrated the greatest target gene enrichment in the ZEB1 transcriptome (miR-181b, let-7f-1-3p, miR-218-2-3p). Compared with control transfectants, 393P cells transfected with antagomiR-181b or antagomiR-let-7f-1 were more migratory and invasive in Boyden chambers (Figure 1, C and D), but cell proliferation and EMT marker levels were not different (Figure $1 \mathrm{E}$ and Supplemental Figure 1C). Conversely, compared with control transfectants, highly metastatic H1299 human lung cancer cells transfected with miR-181b, let-7f-1, or miR-218-2-3p were less migratory in Boyden chambers (Supplemental Figure 1, D and E).

Based on reports that miR-181b functions as a metastasis suppressor $(19,20)$, we performed miR-181b gain-of-function studies on highly metastatic murine (344SQ) and human (H1299) lung adenocarcinoma cells. Following subcutaneous injection into syngeneic, immunocompetent mice, 344SQ_miR-181b cells and $344 \mathrm{SQ}$ _vector cells formed primary tumors of similar size, but the 344SQ_miR-181b tumors generated fewer lung metastases (Figure $1 \mathrm{~F}$ and Supplemental Figure 2A). Compared with 344SQ_vector cells, 344SQ_miR-181b cells formed fewer lung colonies following tail vein injection (Figure $1 \mathrm{G}$ and Supplemental Figure 2B). Compared with empty vector transfectants, miR-181btransfected 344SQ cells and H1299 cells underwent more anoikis in suspension cultures (Figure 1, H and I) and formed fewer colonies in soft agar (Figure 1J) but exhibited no differences in proliferation in monolayer culture (Supplemental Figure 2C). Conversely, compared with scrambled control transfectants, antagomiR-181btransfected 393P cells formed more lung colonies following tail vein injection (Figure $1 \mathrm{~K}$ and Supplemental Figure 2D). Thus, miR-181b functioned as a metastasis suppressor in the context of a ZEB1-driven lung adenocarcinoma model.

We reasoned that if ZEB1 increases metastasis by activating a miR-181b-regulated ceRNA network, then high ZEB1 levels should antagonize the prometastatic activity of antagomiR-181b. To test this idea, we compared the effects of antagomiR-181b on KP cells with high (344SQ, 344P) and low (393P, 307P) ZEB1 levels. Although antagomiR-181b prominently decreased miR-181b levels in both groups (Supplemental Figure 3A), prometastatic effects were observed only in low-ZEB1 KP cells, which demonstrated enhanced cell migration (Figure 2A), reduced anoikis (Figure 2, B and $\mathrm{C}$ ), increased colony formation in soft agar (Figure 2D), and increased miR-181b target gene expression (Figure 2, E and F). Importantly, ectopic ZEB1 expression attenuated the sensitivity of low-ZEB1 tumor cells (393P, 307P, HCC827) to antagomir-181b (Figure 2G and Supplemental Figure 3, B and C). These findings supported further studies to determine whether ZEB1 activates a miR-181b-regulated ceRNA network.

To identify ZEB1-upregulated miR-181b targets that might function as ceRNAs, we queried RNA-Seq data from 393P_ZEB1 cells and 393P_vector cells using a prediction algorithm (www. targetscan.org), which identified 62 genes that contained at least 1 predicted miR-181b-binding site and were increased at least 1.5 - 
A

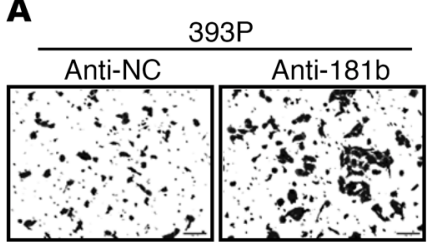

307P

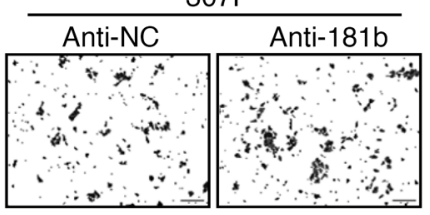

C

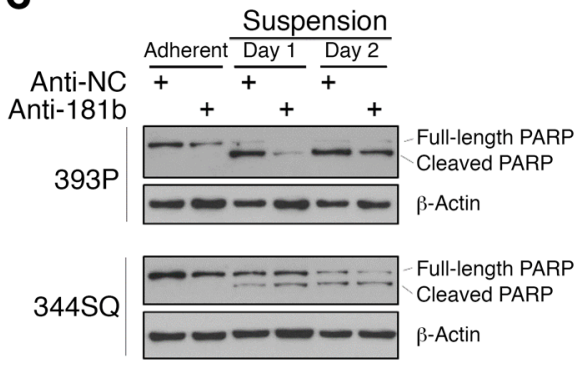

$\mathbf{F}$

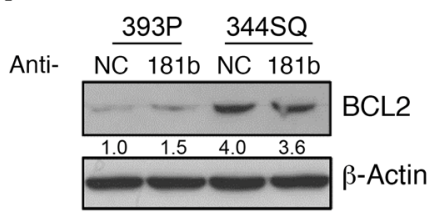

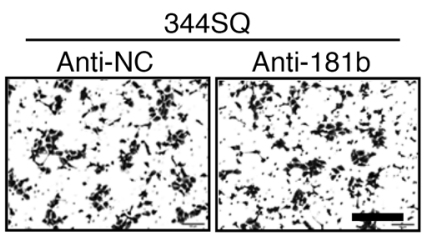

344P

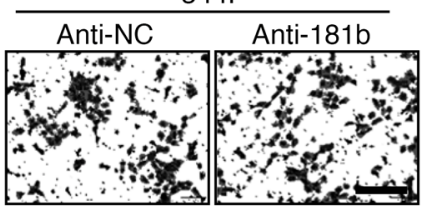

D

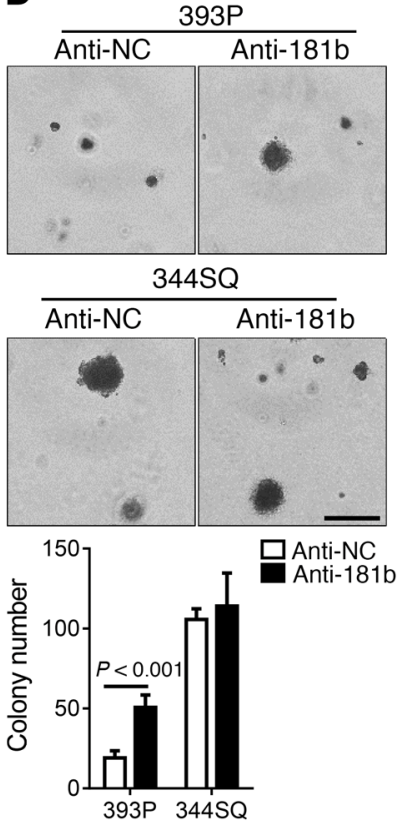

B

$-\bullet \cdot 393 \mathrm{P}+$ anti-NC

- 393P + anti-181b

- $-344 \mathrm{SQ}+$ anti-NC

- 344SQ + anti-181b
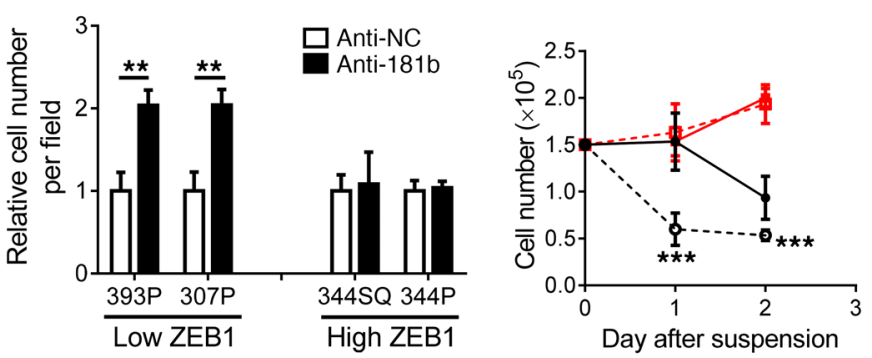

E

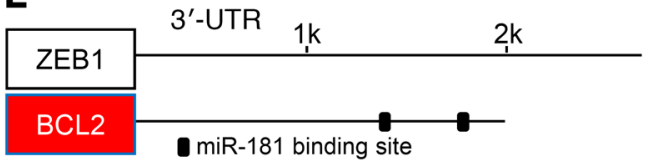

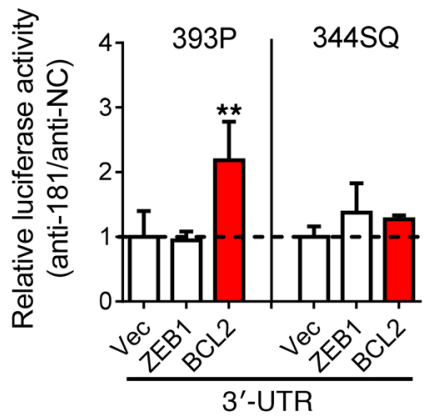

G 393P ZEB1
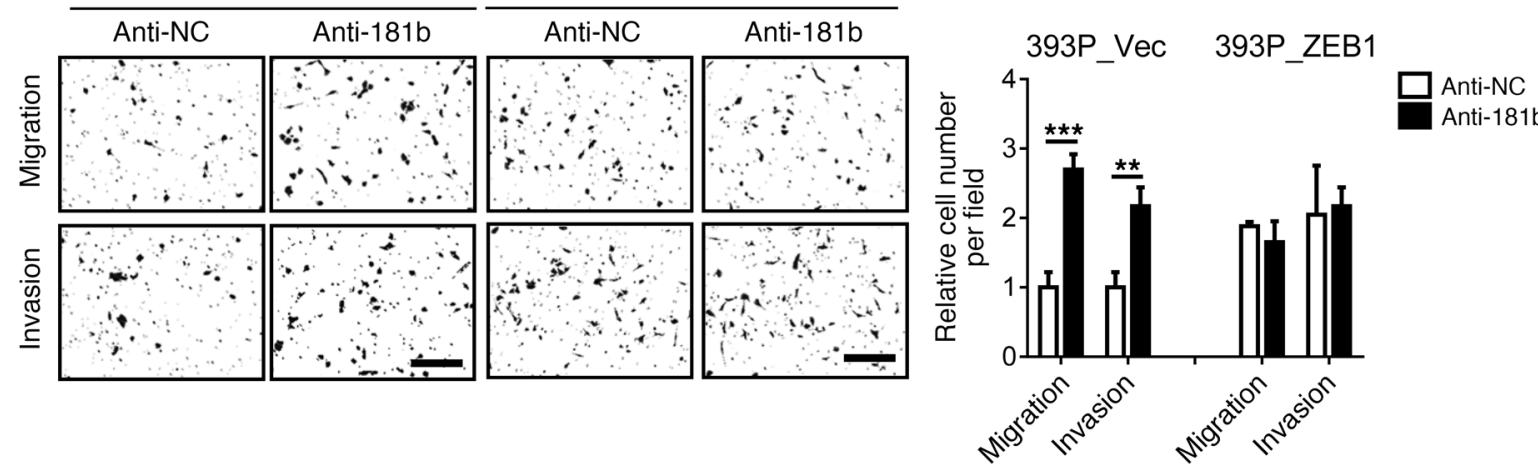

Figure 2. ZEB1 suppresses the prometastatic activity of antagomiR-181b. (A) Boyden chamber assays on KP cells with low endogenous ZEB1 levels (393P and 307P) or high endogenous ZEB1 levels (344SQ and 344P) after transfection with antagomiR-181b or control antagomirs (anti-NC). Migratory cells were photographed (images) and counted (bar graph). Results expressed relative to controls (anti-NC). Scale bars: $200 \mu \mathrm{m}$. (B) Numbers of viable antagomiR181b- and anti-NC-transfected cells seeded on low-adhesion plates and counted 24-48 hours later. $n=4$. (C) Western analysis to detect cleaved PARP in transfectants seeded on adhesive or low-adhesion (suspension) plates. $\beta$-Actin included as loading control. (D) Soft agar colony formation by antagomiR181b- and anti-NC-transfected cells. Colonies were photographed (images) and counted (bar graph). Results expressed relative to controls. Scale bar: $100 \mu \mathrm{m}$. (E) 3'-UTR activity assays of a known miR-181b target, BCL2. 393P and 344SQ cells were transfected with a BCL2 3'-UTR luciferase reporter and antagomiR-181b or anti-NC. Luciferase activity was normalized and expressed as a ratio (antagomiR-181b/anti-NC). ZEB1 3'-UTR included as a negative control. $n=4$. (F) Western analysis of BCL2 levels in 393P and 344SQ cells transfected with antagomiR-181b or anti-NC. The numbers indicate the normalized expression levels of BCL2 protein. (G) Boyden chamber assays on antagomir-181b- and anti-NC-transfected cells. Migratory and invasive cells were photographed (images) and counted (bar graph). Results expressed relative to anti-NC. Scale bars: $200 \mu \mathrm{m}$. ${ }^{* *} P<0.01$ and ${ }^{* * *} P<0.001$. Values are mean \pm SD. $n=3$, unless otherwise indicated. $P$ values, 2-tailed Student's $t$ test. Results were replicated ( $n \geq 2$ experiments). 
fold by ectopic ZEB1 expression (Figure 3A). qPCR analysis of the most highly upregulated miR-181b targets in the KP cell line panel showed that ITGA1 was the only miR-181b target that was differentially expressed between mesenchymal and epithelial KP cells (Supplemental Figure 4A). In The Cancer Genome Atlas human lung adenocarcinoma database (https://gdc.cancer.gov/) $(n=$ 1,016 tumors), ZEB1 and ITGA1 mRNA levels were positively correlated (Supplemental Figure 4B). In 344SQ cells, ITGA1 and miR$181 \mathrm{~b}$ were approximately equimolar ( 300 copies per cell) (Figure $3 \mathrm{~B}$ ), an important determinant of ceRNA functionality (21). ITGA1 contains 3 predicted miR-181b-binding sites in the coding sequence and 1 site in the $3^{\prime}$-untranslated region (3'-UTR) (www. targetscan.org). Relative to empty vector transfectants, miR-181btransfected 344SQ cells had lower activities of reporters containing the ITGA1 3'-UTR but not reporters containing the ITGA1 coding sequence or a mutant ITGA1 3'-UTR lacking the predicted miR-181b site (Figure 3C). A negative control miR (miR-181c) did not suppress ITGA1 3'-UTR activity (Figure 3C). To address whether miR-181b binds to the ITGA1 3'-UTR, we performed R17/ MS2 coat protein-based (MS2-based) RNA immunoprecipitations (22). Compared with MS2 RNA and a negative control miR (miR200b), an MS2-ITGA1 3'-UTR fusion transcript was significantly enriched in miR-181b (Figure 3D). A mutation in the predicted miR-181b-binding site in the ITGA1 3'-UTR abrogated the enrichment (Figure 3D).

ITGA1 encodes $\alpha_{1}$ integrin, which is a component of a heterodimeric collagen receptor $\left(\alpha_{1} \beta_{1}\right)$ that stimulates actin cytoskeletal remodeling and cell motility (23) but has no known role in EMT-driven metastasis. To determine whether it promotes metastasis through a noncoding RNA function, we used shRNAs to generate ITGA1-deficient 344SQ cells, reconstituted them with full-length ITGA1 or with ITGA1 constructs that lack 3 '-UTR or translation start codon sequences, and injected the transfectants subcutaneously or intravenously into syngeneic, immunocompetent mice. Compared with ITGA1-replete cells, ITGA1-deficient 344 SQ cells formed flank tumors that were less metastatic to the lungs following subcutaneous injection (Supplemental Figure 4, C and D) and generated fewer lung colonies following tail vein injection (Supplemental Figure 4, E and F). Lung colonization was partially restored by ITGA1 constructs that lack 3'-UTR or start codon sequences and completely restored by full-length ITGA1 (Figure 3E and Supplemental Figure 5, A and B). Thus, ITGA1 was prometastatic in part through its 3 '-UTR.

To determine whether miR-181b-binding sites in the ITGA1 3'-UTR are required for metastasis, we introduced WT or mutant ITGA1 3'-UTR constructs lacking miR-181b-binding sites into 393P cells, which have relatively low endogenous ITGA1 levels (Supplemental Figure 4A). Compared with control transfectants, 393P cells transfected with the WT ITGA1 3'-UTR had increased migratory activity in Boyden chambers (Figure 3F) and reduced anoikis in suspension cultures (Figure 3G) and generated more lung colonies following tail vein injection (Figure $3 \mathrm{H}$ ), whereas a mutant ITGA1 3'-UTR that lacks the miR-181b-binding site did not increase these metastatic properties (Figure 3, F-H). Similarly, compared with ITGA1-replete cells, ITGA1-deficient H1299 cells had reduced migratory activity in Boyden chambers, and the migratory activity of the ITGA1-deficient H1299 cells was restored by vectors that express WT ITGA1 3 '-UTR but not a mutant ITGA1 3'-UTR that lacks the miR-181b-binding site (Figure 3I). Furthermore, ectopic miR-181b expression diminished the ITGA1 3'-UTR-induced increase in 393P cell migratory activity (Figure 3J), and antagomiR-181b partially rescued the migratory and invasive activities of ITGA1-deficient 344SQ cells (Figure 3K). Thus, the ITGA1 3'-UTR promoted metastasis through its miR181b-binding activity.

ZEB1 upregulates ITGA1 through multiple intermediates. On the basis of the above evidence that ITGA1 is a prometastatic miR181b target, we performed additional studies to elucidate the way in which ZEB1 upregulates ITGA1 expression. Ectopic ZEB1 expression increased RNA polymerase II binding to the ITGA1 gene promoter (Supplemental Figure 6A), indicating that ZEB1 increases ITGA1 gene transcription. However, ZEB1 functions primarily as a transcriptional repressor (24), which led us to reason that ZEB1 increases ITGA1 transcription through intermediates. To test this idea, we examined the kinetics of ITGA1 mRNA upregulation in 393P cells that express ZEB1 under the control of a doxycycline-inducible promoter. ITGA1 mRNA levels increased 72 hours after the initiation of doxycycline treatment, whereas the levels of miR-200b, a ZEB1 target (25), decreased within 24 hours (Supplemental Figure 6B). To determine whether the delay was the result of ZEB1-induced epigenetic regulation of the ITGA1 genomic locus, we used a prediction algorithm (http://www. urogene.org/methprimer/) and identified a CpG island in the ITGA1 gene locus $(+186$ to $+400:+1$ refers to the first nucleotide of the 5 -UTR) that bisulfite sequencing revealed to be more methylated in epithelial than in mesenchymal KP cells and that was demethylated in response to ectopic ZEB1 expression (Figure $4 \mathrm{~A}$ ). Treatment with the DNA methyltransferase inhibitor 5azacytidine increased ITGA1 mRNA levels in epithelial (393P, 307P, HCC827) but not mesenchymal (344SQ, 393P_ZEB1) lung cancer cells (Figure 4B and Supplemental Figure 6C). Relative to empty vector transfectants, ZEB1-transfected 393P cells had higher mRNA levels of ten-eleven translocation 2 gene (TET2) and lower levels of the DNA methyltransferases DNMT1 and DNMT3B (Figure 4C). In the KP cell line panel, mesenchymal differentiation status was positively correlated with TET2 levels and negatively correlated with DNMT1 and DNMT3B levels (Supplemental Figure 6C). In TET2 and DNMT1/3B gain- and loss-offunction studies, ITGA1 levels were upregulated by TET2 (Figure 4, D and E) but not DNMT1 or DNMT3B (Supplemental Figure 6, D-F). Thus, ZEB1 upregulated TET2 to relieve an epigenetic repression of ITGA1.

Because $\mathrm{CpG}$ island demethylation facilitates transcription factor binding to promoter elements (26), we examined whether ITGA1 is a target of ZEB1-upregulated transcription factors. Relative to empty vector transfectants, ZEB1-transfected 393P cells had higher ITGA1 promoter reporter activity (Supplemental Figure 6G), which was attenuated by mutation of an ARNT-binding site but not predicted binding sites for other transcription factors (Figure 4F). ARNT bound to the ITGA1 promoter in chromatin immunoprecipitation assays (Figure 4G). ITGA1 mRNA levels (Figure 4H) and ITGA1 promoter reporter activity (Figure 4I) were lower in ARNT siRNA-transfected cells than in scrambled controls. ARNT mRNA and protein levels were higher in mesenchy- 
A

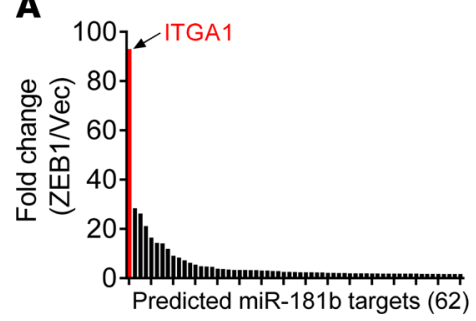

B

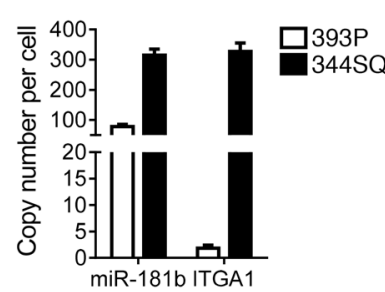

D
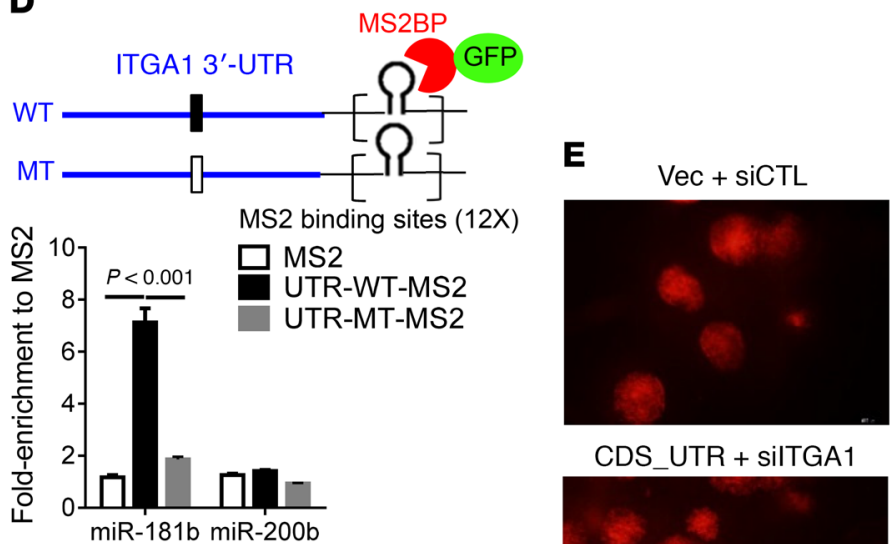

CDS_UTR + SilTGA 1

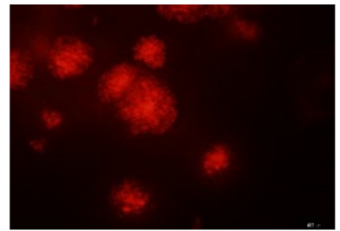

$\mathbf{F}$
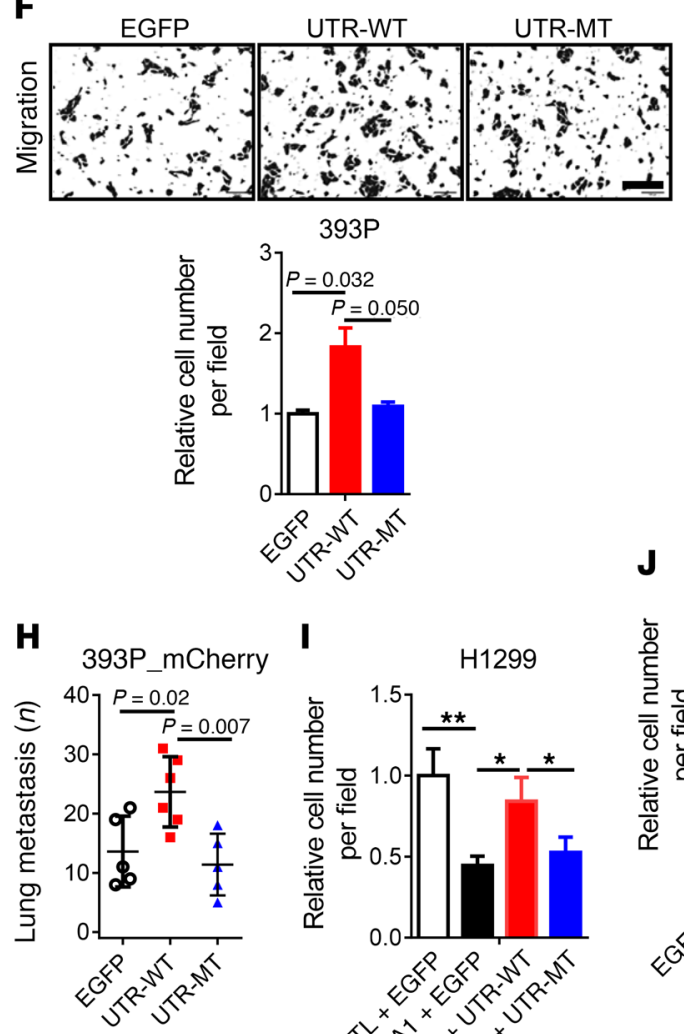

I
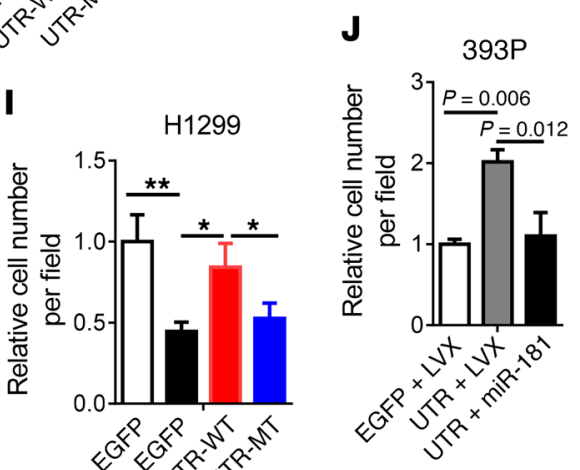

K
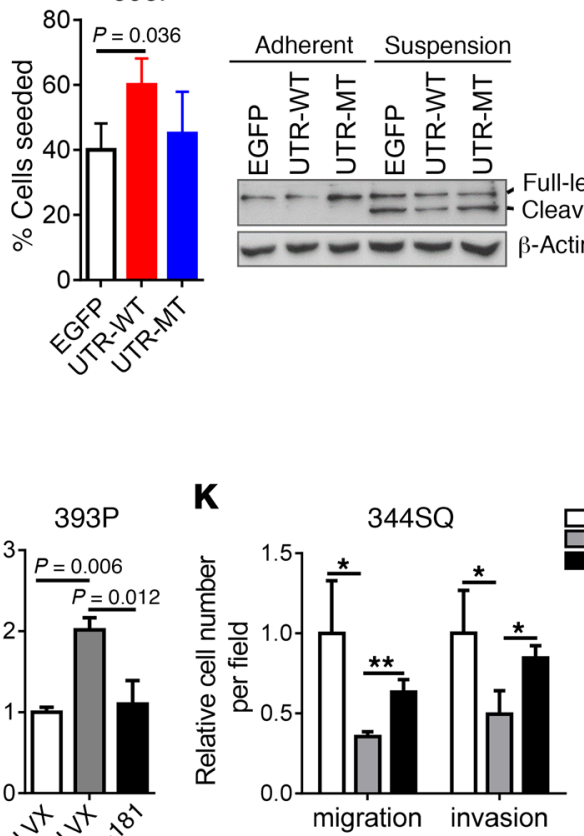

344SQ RFP

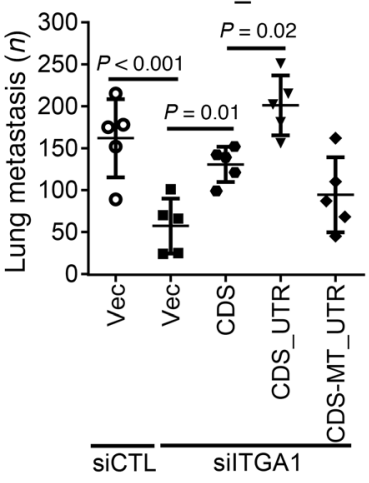

Adherent Suspension

$0 \sum_{1} \sum_{1}$

$0 \sum_{1}^{5} \sum_{i}$

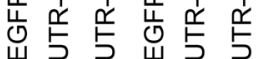

Ш

Cleaved PARP

$-\infty-\infty-\infty$-Actin

EGFP

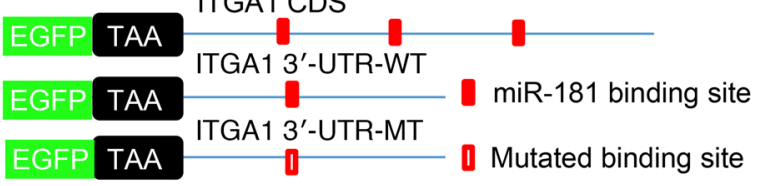

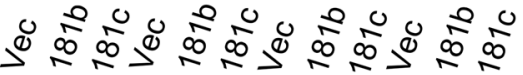
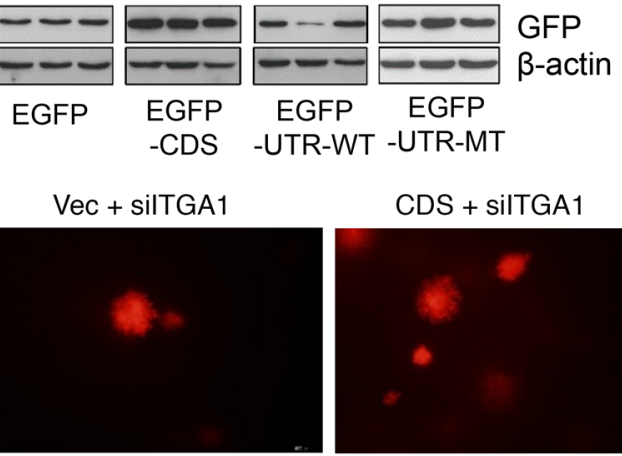

CDS + silTGA1

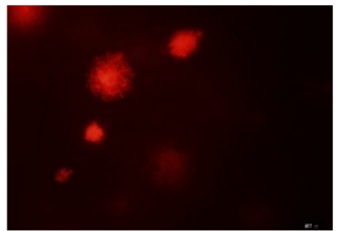

CDS-MT_UTR + silTGA1

$400 \mu \mathrm{m}$

$$
\text { . }
$$

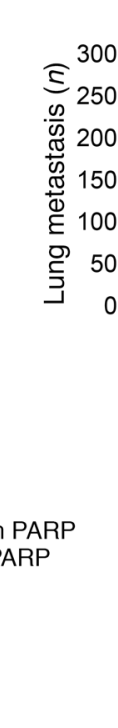


Figure 3. ITCA1 3'-UTR promotes metastasis through its miR-181b-binding site. (A) RNA-Seq analysis of predicted miR-181b targets in 393P_ZEB1 cells and 393P_vector cells expressed as a ratio (ZEB1/Vec). (B) miR-181b and ITCA1 mRNA copy numbers. (C) EGFP reporters fused to ITCA1 coding sequence (CDS) or $3^{\prime}$-UTR containing wild-type (WT) or mutated (MT) miR-181b-binding sites. Western analysis (bottom) of EGFP in 344SQ cells cotransfected with EGFP reporters and miR-181b, miR-181c, or vector (Vec). (D) Constructs containing MS2 binding sites (12X) fused downstream of a WT (UTR-WT-MS2) or mutated (UTR-MT-MS2) ITCA1 3'-UTR. MS2-based RIP (bottom). MS2-UTR-associated miR-181b and miR-200b (negative control) quantified as fold enrichment relative to MS2. (E) Fluorescence microscopic images of lung metastases in mice injected with the 344SQ_RFP transfectants (see Supplemental Figure 5, A and B). Scatter plot: numbers of lung metastases per mouse (dots). (F) Boyden chamber migration assays. 393P cells transfected with ITCA1 3'-UTR (WT or MT) or EGFP. Migratory cells were photographed (images) and counted (bar graph). Scale bar: $200 \mu \mathrm{m}$. (G) Numbers of viable 393P transfectants on low-adhesion plates (bar graph). Western blotting (gels) to detect cleaved PARP in transfectants on adhesive or low-adhesion (suspension) plates. (H) Numbers of lung metastases in mice injected by tail vein with mCherry-labeled 393P cells transfected with ITCA1 3'-UTR (WT or MT) or control (EGFP). (I) Boyden chamber assays to quantify migratory $\mathrm{H} 1299$ cells cotransfected with a shRNA (shCTL or shITCA1) and a vector that expresses EGFP or ITCA1 3'-UTR (WT or MT). ${ }^{*} P<0.05$ and ${ }^{*} P=0.005$. (J) Boyden chamber assays. 393P cells cotransfected with ITCA1 3'-UTR or EGFP and miR-181b or vector (LVX). (K) Boyden chamber assays. 344SQ cells cotransfected with ITCA1 siRNA or control (siCTL) and antagomiR-181b or anti-NC. ${ }^{*} P<0.05$ and ${ }^{*} P<0.01$. Values are mean $\pm \mathrm{SD} . n=3$, unless otherwise indicated. $P$ values, Dunnett's test. Results were replicated ( $n \geq 2$ experiments).

mal than in epithelial KP cell lines (Supplemental Figure 6, H and I) and in 393P_ZEB1 cells than in 393P_vector cells (Supplemental Figure 6J). Thus, ZEB1 upregulated ITGA1 through ARNT.

ZEB1 silences miRs that target key drivers of EMT and metastasis $(5,11,12)$. To determine whether ITGA1 is a target of ZEB1silenced miRs, we used a prediction algorithm (www.targetscan. org) to query the ITGA1 3'-UTR. Binding sites for multiple miRs were identified, including sites for miR-148a, which is silenced by ZEB1 (12). In reporter assays, ITGA1 3'-UTR activity was suppressed by cotransfection of miR-148a or miR-181b (Figure 4J), and a mutation in the miR-148a-binding site reversed the suppression (Figure 4J). ITGA1 levels were lower in miR-148a-transfected tumor cells than in control transfectants (Figure $4 \mathrm{~K}$ and Supplemental Figure 6K), and miR-148a levels were lower in 393_ZEB1 cells than in 393P_vector cells (Supplemental Figure 6L). Collectively, these findings suggest that ZEB1 upregulated ITGA1 through TET2, ARNT, and miR-148a (Figure 4L).

ITGA1 competes with ADCY9 for binding to miR-181b. To identify protein-coding genes that compete with ITGA1 for binding to miR-181b, we mined the RNA-Seq data from 393P_ZEB1 cells and 393P_vector cells and identified 32 predicted miR-181b targets that are approximately equimolar with ITGA1 on the basis of having RPKM (reads per kilobase of transcript per million mapped reads) values greater than 0.5 -fold and less than 2 -fold that of ITGA1 (Figure $5 \mathrm{~A})$. Relative to their levels in scrambled controls, 4 of the 32 genes in antagomiR-181b-transfected 393P cells were upregulated more than 2-fold and were significantly higher (Figure 5A, indicated in red). Two of the 4 genes (ADCY9 and ONECUT2) were expressed at significantly higher levels in mesenchymal than in epithelial $\mathrm{KP}$ cell lines (Supplemental Figure 7A). Activities of reporters driven by the 3 '-UTRs of ADCY9 or ONECUT2 were lower in miR-181b- transfected 344SQ cells than in negative control transfectants, and loss of the miR-181b-binding sites in the ADCY9 and ONECUT2 3'-UTRs ablated the miR-181b-induced suppression of both reporters (Figure 5B), which is consistent with a prior report on ADCY9 (27).

To determine whether predicted miR-181b targets identified from the above screen can compete with ITGA1 for binding to miR-181b, we subjected ITGA1-deficient and -replete 344SQ cells to Argonaute 2 (AGO2) immunoprecipitation assays. ITGA1 depletion led to a significant increase in the incorporation of ADCY9 but not TGFBR3, ONECUT2, or BHLHE41 into the RNA-induced silencing complex (Figure 5C). Incorporation of ADCY9 into the RNA-induced silencing complex was reduced by ectopic expression of the ITGA1 3'-UTR but not a mutant ITGA1 3'-UTR that lacks the miR-181b-binding site (Figure 5D).

On the basis of the above findings, we examined whether ITGA1 regulates ADCY9 expression through competition for binding to miR-181b. ADCY9 mRNA levels were higher in ITGA1 3'-UTR-transfected 393P cells than in controls, whereas a mutant ITGA13'-UTR that lacks the miR-181b-binding site did not increase ADCY9 levels (Figure 5E). Conversely, ADCY9 levels were lower in ITGA1 3'-UTR siRNA-transfected 344SQ cells than in scrambled controls and were not rescued by cotransfection of ITGA1 coding sequences (Supplemental Figure 7, B and C). ADCY9 3'-UTR reporter activity was higher in ITGA13'-UTR-transfected 393P cells than in controls and was reduced by loss of the miR-181b-binding sites in either $3^{\prime}$-UTR (Figure 5F). Conversely, relative to scrambled controls, ITGA1 3'-UTR siRNA-transfected 344SQ cells had lower activity of a WT ADCY9 3'-UTR reporter but not a mutant ADCY9 3'-UTR reporter lacking the miR-181b-binding sites (Supplemental Figure 7D). To determine whether ZEB1-induced upregulation of ADCY9 is ITGA1-dependent, we depleted ITGA1 from 393P_ZEB1 cells and found that ITGA1 depletion suppressed ZEB1-induced ADCY9 mRNA and protein levels (Supplemental Figure 7, E and F).

On the basis of the above evidence that ITGA1 regulates ADCY9 levels through competition for miR-181b, we reasoned that ADCY9 and ITGA1 could interact bidirectionally through this competition. To address this possibility, we first depleted ADCY9 from 344SQ cells and found that, relative to control oligomer-transfected cells, ADCY9 siRNA-transfected cells had reduced ITGA1 mRNA levels (Supplemental Figure 7G). To determine whether ADCY9 upregulates ITGA1 levels through competition for miR-181b, we ectopically expressed the ADCY9 3'-UTR in 393P cells and found that ITGA1 mRNA levels were higher in ADCY9 3'-UTR-transfected cells than in enhanced green fluorescent protein-transfected (EGFP-transfected) controls, whereas a mutant ADCY9 3'-UTR that lacks miR-181b-binding sites did not have this effect (Supplemental Figure 7H). Moreover, in the KP cell line panel, ITGA1 and ADCY9 levels were positively correlated (Supplemental Figure 7I). Thus, ITGA1 and ADCY9 are components of a miR-181b-regulated ceRNA network.

To determine whether these findings are broadly applicable to human cancer, we mined RNA-Seq data from The Cancer Genome Atlas, the largest publicly available cohort of human cancer transcriptomes. Lung adenocarcinomas $(n=541)$ were grouped on the basis of high or low expression (defined as top and bottom quartiles, respectively) of ITGA1 and ADCY9. ADCY9 levels were significantly higher in the ITGA1-high group 
A
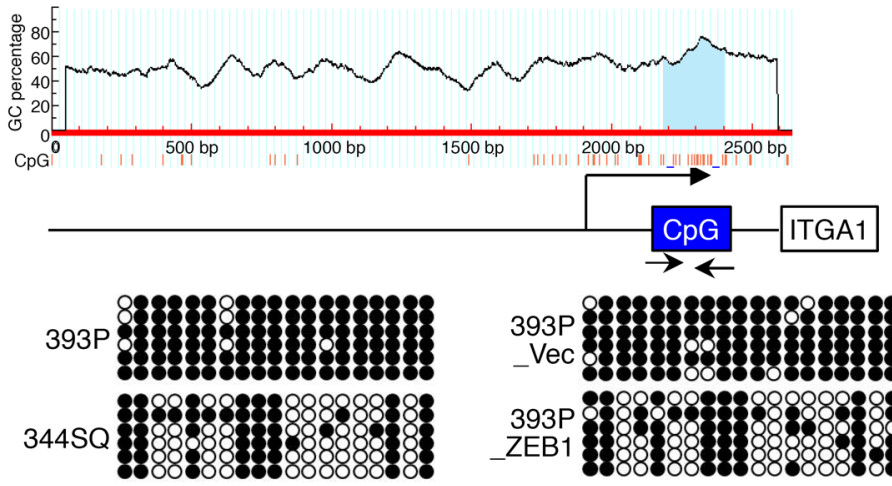

D

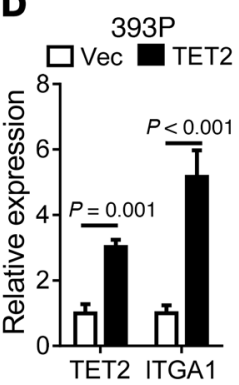

E

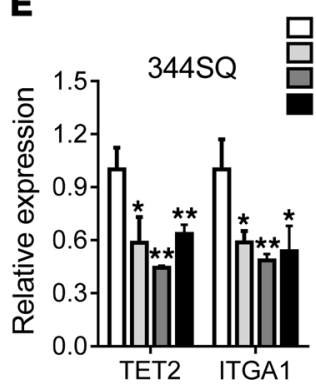

$\mathbf{F}$
B

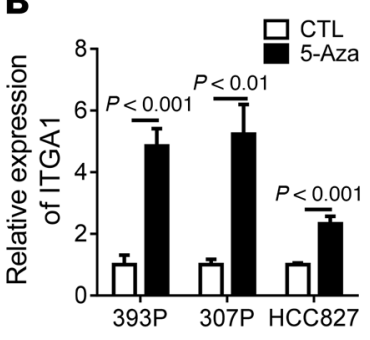

$393 \mathrm{P}$

_Vec

$393 \mathrm{P}$

ZEB1
C

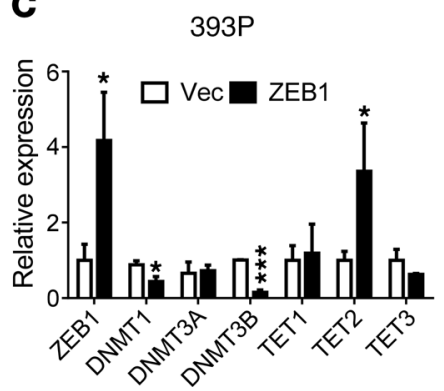

G
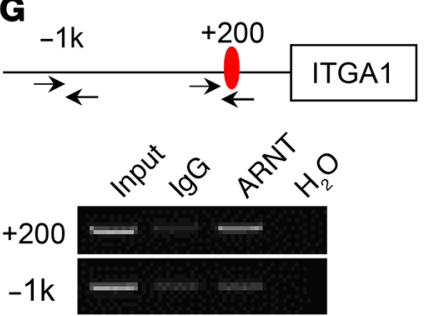

H

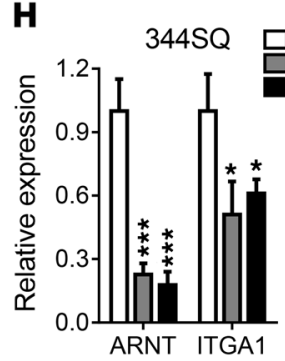

Relative luciferase activity

ITGA1 promoter

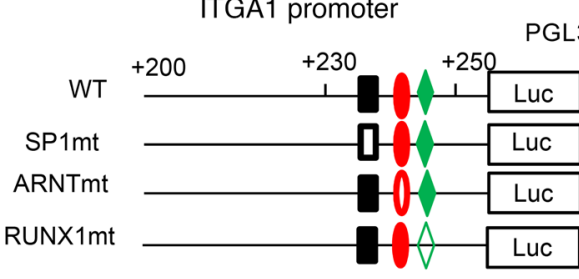

\begin{tabular}{llllll}
0.0 & 0.1 & 0.2 & 0.3 & 0.4 & 0.5 \\
\hline & & $\square$ Vec & ZEB1
\end{tabular}

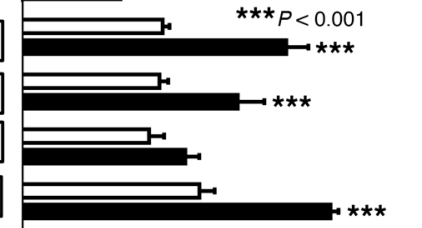

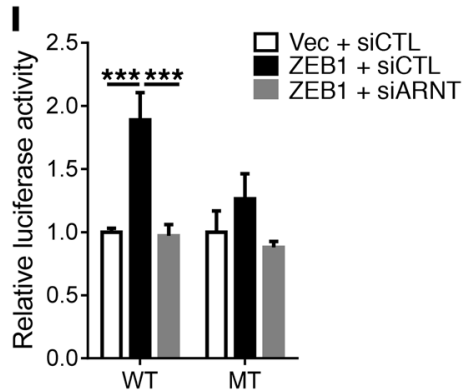

J Luciferase ITGA1 3'-UTR
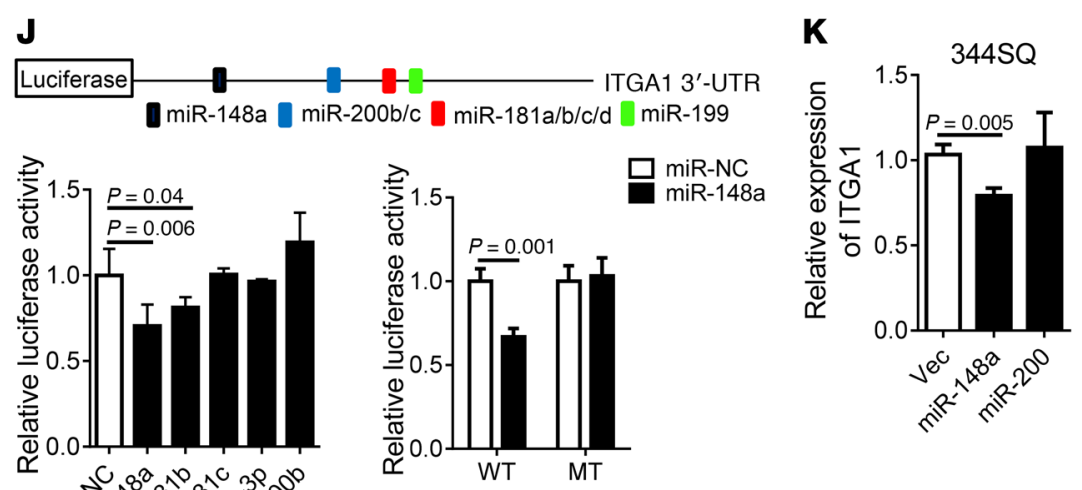

$\mathbf{L}$

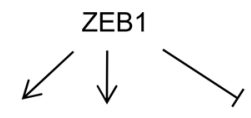
ARNT TET2 miR-148a

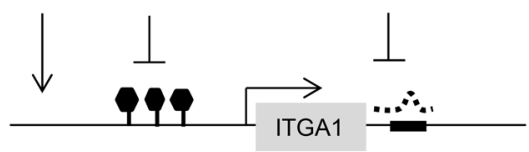

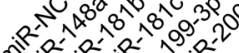


Figure 4. ZEB1 upregulates ITCA1 expression through intermediates. (A) Top: locations of GC repeats (red hash marks) and CpG island (blue shading). Middle: ITCA1 promoter. First nucleotide in $5^{\prime}-$ UTR $(+1)$ and CpG island (CpC). Bottom: bisulfite sequencing. Methylated (black circles) and unmethylated (white circles) CpG repeats (rows) in replicate reactions (columns). (B) QPCR of mRNAs after vehicle (CTL) or 5-azacytidine (5-Aza) treatment. (C) qPCR of mRNAs in 393P_ZEB1 cells (ZEB1) and 393P_vector cells (Vec). ${ }^{*} P<0.05$ and ${ }^{* *} P<0.001$. (D) qPCR of mRNAs in 393P transfectants. Vec, empty vector. (E) qPCR of mRNAs in $3445 Q$ transfectants (scrambled [shCTL] or TET2 [shTET2] shRNAs). ${ }^{*} P<0.05$ and ${ }^{* *} P<0.01$. (F) Schema, ITCA1 promoter constructs; SP1-, ARNT-, and RUNX1-binding sites (squares, ovals, and diamonds, respectively). Solid, WT; open, mutated. Bar graph: luciferase activity in 393P cells cotransfected with ZEB1 or empty (Vec) expression vectors and empty ( $\mathrm{CLL} 3$ ) or ITCA1 promoter reporter. ${ }^{* * *} P<0.001$. (G) ITCA1 chromatin immunoprecipitation assays before (input) or after immunoprecipitation with anti-ARNT (ARNT) or IgG. Gel: PCR of ARNT-binding site (+200) or control ITCA1 promoter locus (-1k) or water. Arrows, PCR primer locations. (H) qPCR of mRNAs in 344SQ transfectants (siCTL or ARNT siRNAs). ${ }^{*} P=0.02$ and ${ }^{* *} P<0.001$. (I) Luciferase activity in 393P cells cotransfected with ZEB1 or empty (Vec) expression vectors, siARNT or siCTL, and an ITCA1 promoter reporter (WT or mutant [MT] ARNT-binding sites). ${ }^{* *} P<0.001$. (J) Top: ITCA1 3 '-UTR reporter. Bottom left: luciferase activity in $3445 Q$ cotransfectants (reporter and miRs or negative control miR-NC). Bottom right: luciferase activity in 344SQ cotransfectants (miR-148a or miR-NC and ITCA1 3'-UTR reporter containing miR-148a-binding sites [WT or MT]). (K) qPCR of mRNAs in 344SQ cells transfected with miRs or empty vector (Vec). (L) Working model. Values are mean \pm SD. $n=3$. $P$ values, 2-tailed Student's $t$ test and Dunnett's test for 2-group and more-than-2-group comparisons, respectively). Results were replicated ( $n \geq 2$ experiments).

than in the ITGA1-low group (Figure 5G), and ITGA1 levels were significantly higher in the ADCY9-high group than in the ADCY9-low group (Figure 5H). Moreover, by pairwise comparisons, ZEB1, ITGA1, and ADCY9 were positively correlated with each other in 16 of 32 different cancer types examined (Figure 5I and Supplemental Table 1).

ADCY9 mediates ZEB1-driven metastasis. ADCY9 encodes 1 of 9 transmembrane adenylyl cyclase (AC) protein isoforms (AC1-9) that catalyze the synthesis of cAMP from ATP and are regulated primarily by heterotrimeric $G$ protein-coupled receptors (28). As a second messenger molecule, cAMP binds and activates multiple effector proteins that have been studied extensively in cancer (28), but whether AC proteins regulate metastasis remains unclear.

To address this question, we first quantified cAMP levels in the KP cell line panel. cAMP levels were higher in mesenchymal KP cells than in epithelial KP cells (Supplemental Figure 8A), and they were higher in 393P_ZEB1 cells than in 393P_vector cells (Supplemental Figure 8B). Next, we treated 393P cells with the membrane-permeable cAMP analog 8-bromo-cAMP or the AC activator forskolin (Supplemental Figure 8C). Both treatments increased 393P cell migration and invasion in Boyden chambers (Figure 6A and Supplemental Figure 8D). These findings show that ZEB1 increased cAMP production, which enhanced the metastatic properties of tumor cells.

On the basis of the above findings, we examined whether ZEB1-driven metastasis requires ADCY9. Relative to scrambled control transfectants, ADCY9 siRNA-transfected 344SQ cells (Figure 6B) had lower cAMP levels (Figure 6C), were less migratory and invasive in Boyden chambers (Figure 6D), exhibited more anoikis in suspension cultures (Figure 6E), formed fewer colonies in soft agar (Figure 6F), and generated fewer lung colonies after tail vein injection (Figure 6G and Supplemental Figure 8E). Conversely, compared with empty vector transfectants, 393P cells that ectopically express ADCY9 (Figure 6H) had higher cAMP levels (Figure 6I) and were more migratory (Figure 6J). To determine whether ADCY9 is sufficient to reverse miR-181b's metastasis suppressor activity, we restored ADCY9 levels in 344SQ and H1299 cells that ectopically express miR-181b and showed that, in Boyden chambers, migratory activity was higher in ADCY9restored cells than in empty vector-transfected cells (Supplemental Figure 8F). To confirm that ADCY9 mRNA promoted tumor cell migration through its protein product rather than its 3 '-UTR, we reconstituted ADCY9-deficient 344SQ cells with protein-c oding or 3'-UTR sequences of ADCY9 (Supplemental Figure 8G) and showed that, in Boyden chambers, the migratory activity of ADCY9deficient cells was restored to a greater extent by protein-coding sequences than by $3^{\prime}$-UTR sequences (Supplemental Figure 8H).

To determine whether ADCY9 is the only AC isoform through which ZEB1 activates cAMP production, we quantified the mRNA levels of all 9 transmembrane ADCY isoforms. Relative to 393P_vector cells, 393P_ZEB1 cells had higher mRNA levels of ADCY3, ADCY7, and ADCY9 (Supplemental Figure 9A). Compared with scrambled control transfectants, ADCY7 siRNA-transfected 344SQ cells (Supplemental Figure 9B) had lower cAMP levels (Supplemental Figure 9J) and reduced migratory activity in Boyden chambers (Supplemental Figure 9C) but exhibited no change in cell proliferation (Supplemental Figure 9D). In contrast, ADCY3 siRNA-transfected 344SQ cells (Supplemental Figure 9B) had no detectable change in cAMP levels (Supplemental Figure 9C) or 344SQ cell migration (Supplemental Figure 9F) or proliferation (Supplemental Figure 9G). Thus, ZEB1 activates cAMP production and promotes $344 \mathrm{SQ}$ cell migration through ADCY7 and ADCY9.

On the basis of the above evidence that ADCY9 promotes metastasis in a ZEB1-driven lung adenocarcinoma model, we examined the prognostic value of ADCY9 in human lung adenocarcinomas. For this purpose, we probed a compendium of clinically annotated human lung adenocarcinomas $(n=1,586)$ with a signature of 1,421 genes that were differentially expressed (601 up, 820 down; $P<0.01$ by $t$ test, fold change $>1.4$ ) in ectopic ADCY9-expressing and empty vector-transfected 393P cells (Supplemental Table 2). qPCR analysis confirmed that 19 of 20 genes sampled were differentially expressed in 393P_ADCY9 cells and 393P_vector cells (Supplemental Figure 10A). Enrichment analysis showed that the differentially expressed genes have diverse gene functions (Supplemental Table 2) but none related to EMT, which we confirmed by qPCR analysis of EMT markers (Supplemental Figure 10, B and C). Using a " $t$ score" approach described previously (29), we identified a manifestation of the 1,421-gene signature in human lung adenocarcinomas that was correlated with a significantly shorter duration of survival (log-rank $\left.P=7.6 \times 10^{-8}\right)$ (Supplemental Figure 10D).

\section{Discussion}

We have identified a ceRNA network that plays a key role in ZEB1driven lung adenocarcinoma metastasis. ITGA1 and ADCY9 competed for binding to miR-181b, and ZEB1 upregulated ITGA1 


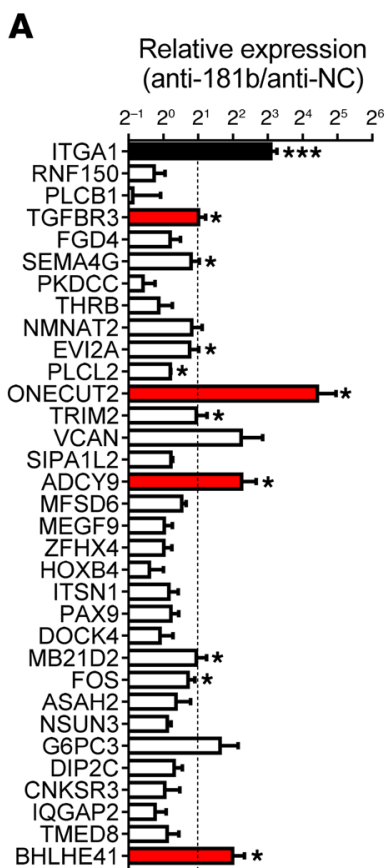

D

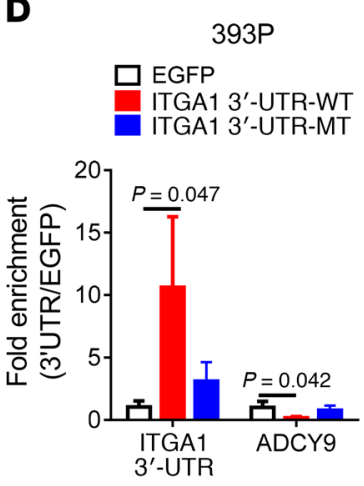

G

ITGA1 ADCY9
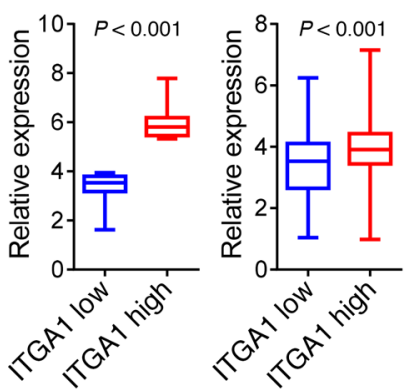

B

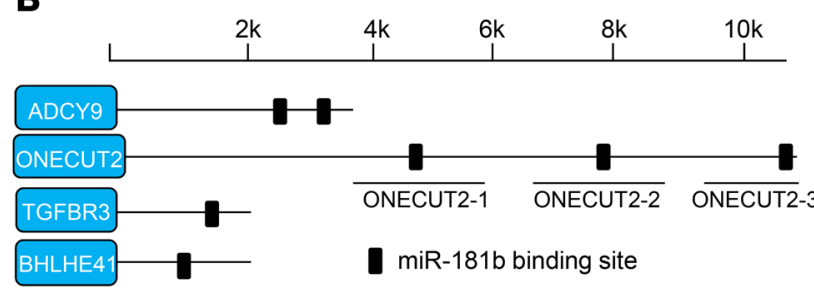

C

$344 S Q$

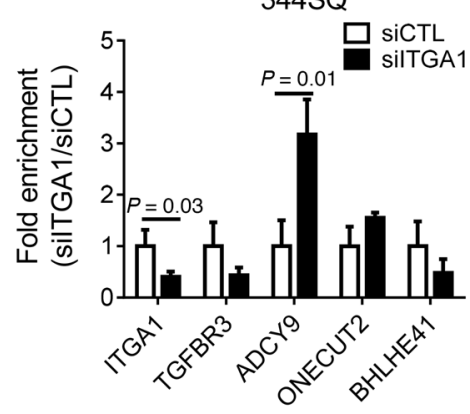

E 393P

F 393P
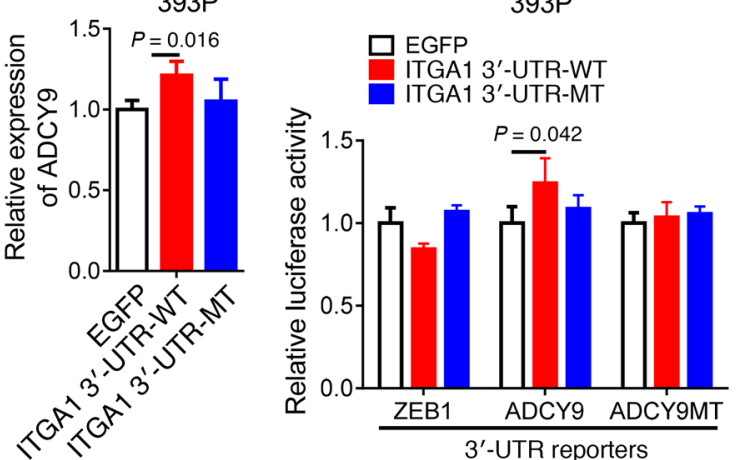

H ADCY 9

ITGA1
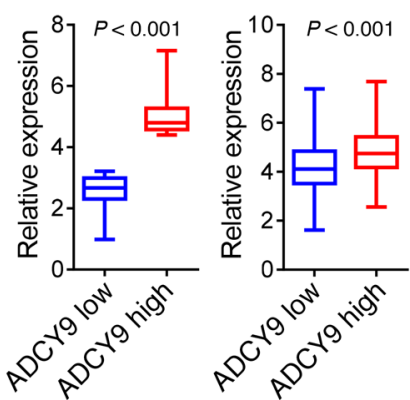

I

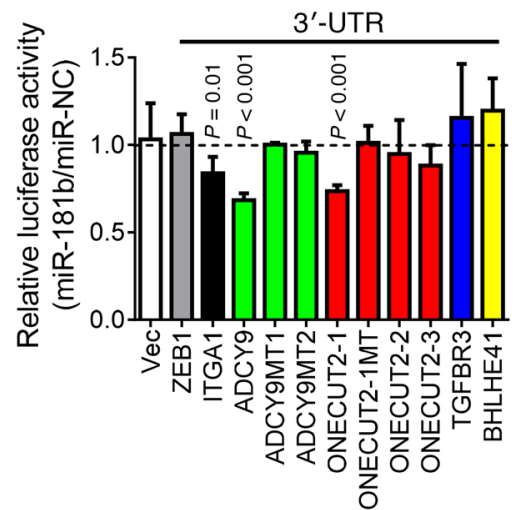

Pearson's correlation $(t)$ negative positive $-6-4-202446$

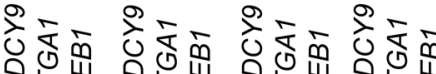

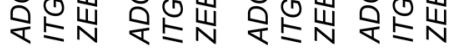
ADCY9 ITGA1 ZEB1
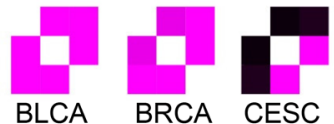

ADCY9

ITGA1

ZEB1
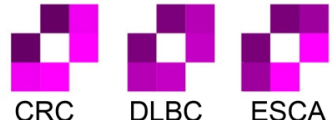

$A D C Y 9$

ITGA1

ZEB1

ADCY9

ITGA1

ZEB1

CHOL
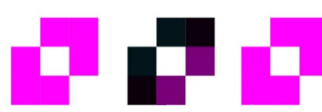

GBM HNSC $\mathrm{KICH} \quad \mathrm{KIRC}$

KIRP LAML LGG LIHC

$A D C Y$

ITGA1
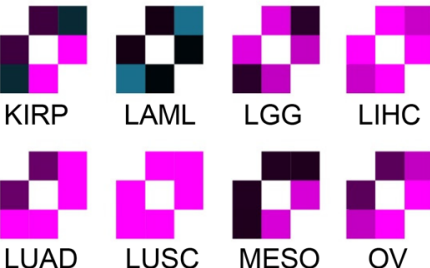

$A D C Y 9$

ITGA1

ZEB1
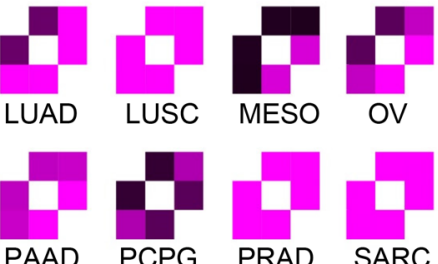

ADCY9

ITGA1

ZEB1
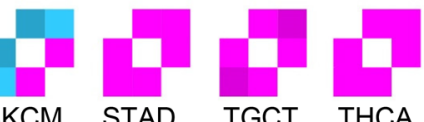

ADCY9

ITGA1

ZEB1
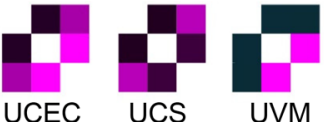
Figure 5. ITCA1 and ADCY9 are miR-181b-regulated ceRNAs. (A) qPCR of mRNAs in 393P transfectants (antagomiR-181b [anti-181b] or control [anti$\mathrm{NC}]$ ). Levels of 32 predicted miR-181b targets that were approximately equimolar with ITCA1 in 393P_ZEB1 cells expressed as a ratio (anti-181b/anti-NC). Red, significantly upregulated more than 2-fold. ${ }^{*} P<0.05$ and ${ }^{* *} P<0.001$. (B) Schema, 3'-UTRs of ADCY9, ONECUT2, TCFBR3, and BHLHE41 with locations of predicted miR-181b-binding sites. Bar graph: luciferase activity in 344 SQ cells cotransfected with miR-181b or control (miR-NC) and a 3'-UTR reporter with WT or mutant (MT) miR-181b-binding sites. ONECUT2 reporters with 3'-UTR portions (ONECUT2-1, -2, and -3). (C) AGO2 RIP performed on lysates from $344 \mathrm{SQ}$ transfectants (ITCA1 siRNA [silTGA1] or control [siCTL]). ACO2-associated ITCA1, TGFBR3, ONECUT2, and ADCY9 mRNAs normalized on the basis of IgC control and expressed as fold enrichment relative to siCTL. (D) AC02 RIP on 393P cells transfected with WT or mutant (MT) ITCA1 3'-UTR or EGFP. ACO2-associated ITCA1 3'-UTR and ADCY9 mRNA normalized on the basis of IgG control and expressed as fold enrichment relative to EGFP transfectants. (E) qPCR of ADCY9 mRNA in 393P cells transfected with ITCA1 3'-UTR (WT or MT) or EGFP. (F) Luciferase activities in 393P cells cotransfected with ADCY9 3'-UTR reporters and ITCA1 3'-UTR (WT or MT) or EGFP. ZEB1 3'-UTR reporter included as negative control. (G and H) ITCA1 and ADCY9 levels in human lung adenocarcinomas $(n=541)$ in The Cancer Cenome Atlas (TCGA) as groups (top and bottom quartiles). (I) Pan-cancer analysis examining correlations between ZEB1, ITCA1, and ADCY9 mRNA levels for each of 32 different cancer types in TCGA. See Methods (Statistics section) for list of tumor types and sample sizes. Values are mean \pm SD. $n=3$, unless otherwise indicated. $P$ values, 2-tailed Student's $t$ test and Dunnett's test for 2-group and more-than-2-group comparisons, respectively. Results were replicated ( $n \geq 2$ experiments)

to activate a miR-181b-regulated ceRNA network that increased metastasis through AC9 (Figure 7). Despite potently inhibiting metastasis, inactivation of the prometastatic ceRNA network did not modulate the expression of EMT markers, suggesting that ZEB1 drives metastasis and EMT through distinct signaling pathways. In human lung cancers, high levels of miR-181b had good-prognostic value, ITGA1 and ADCY9 were positively correlated, and an AC9-activated transcriptomic signature had poorprognostic value. Thus, the ceRNA network identified here may negatively influence clinical outcome in lung cancer patients.

Although it is well established that ZEB1 downregulates miRs by silencing the promoters of the miRs themselves or the expression of transcription factors that drive miR expression $(4,11,12)$, the results of the present study show that ZEB1 can inactivate miRs by upregulating ceRNAs. Furthermore, they build on a growing body of evidence that miRs are key mediators of EMT-TFs. For example, mutual antagonism between the transcription factors and miRs (e.g., ZEB1 and miR-200, SNAIL, and miR-34a) regulates tumor cells' ability to shift reversibly between epithelial and mesenchymal states (3). Additionally, ZEB1 silences the expression of miRs that suppress tumor cell stemness by targeting the stem cell factors SOX2 and KLF4 (e.g., miR-203 and miR-183); that inhibit tumor cell motility by targeting genes that promote the remodeling of actin-based cytoskeletal structures (e.g., miR-34a); and that block vesicle trafficking toward the leading edge of the cell by targeting a Golgi scaffolding protein that promotes the formation of a compact, polarized Golgi organelle (e.g., miR-148a, miR-206, and miR-200) $(4,11,12)$. Additional studies are warranted to determine the degree to which ceRNA networks extend the prometastatic activities of EMT-TFs.

Numerous types of noncoding RNAs (e.g., long noncoding RNAs, small noncoding RNAs, pseudogenes, circular RNAs) are considered to be ideally suited for ceRNA activities (30). Classic examples include the PTENP1 pseudogene, which competes with its cognate gene, the tumor suppressor PTEN, for binding to multiple miRs in tumor cells with an intact PTEN locus, and the HULC long noncoding RNA, which competes with the catalytic subunit of cAMP-dependent protein kinase (PKA) for binding to miR-372 in hepatocellular carcinoma $(13,31)$. In addition, there is a growing list of protein-coding RNAs that have validated ceRNA activities, including PTEN, which competes with other proteincoding mRNAs (CNOT6L, VAPA, ZEB2, VCAN, and others) for binding to miRs and shares at least $2 \mathrm{miR}$ binding sites with each of these genes, which underlies its potency as a ceRNA $(22,32)$. However, a single miR binding site can be sufficient for a proteincoding gene to have ceRNA functionality; for example, COL1A1 and CD44 compete for binding to miR-328 (33), and the results of the present study show that ITGA1 competes with ADCY9 for binding to miR-181b. Furthermore, it is conceivable that PTEN and ZEB1, which share miR-200b-binding sites, could function as ceRNAs and that PTEN could thereby modulate the ZEB1driven ceRNA network described here.

In many cases, the biological effects that mRNAs exert through their protein coding and noncoding functions are quite distinct and can be either complementary or antagonistic (30). Similarly, the biological functions of $\alpha_{1} \beta_{1}$ integrin (23) are distinct from the noncoding functions of ITGA1 observed here. In the case of ITGA1, dual protein coding and noncoding functionality is likely given that metastasis-prone tumor cells had detectable levels of $\alpha_{1}$ integrin protein, and reconstitution of ITGA1-deficient tumor cells with the ITGA1 3 '-UTR only partially reversed the effects of ITGA1 depletion. The way in which cells regulate mRNA participation in protein coding and/or noncoding functions remains unclear and will be an important direction of future research.

The findings presented here build on a growing body of evidence that EMT- and cAMP-dependent pathways are tightly interwoven. The regulatory (PRKAR1A) and catalytic (PRKACB) subunits of PKA are targets of miR-200c, which is silenced by ZEB1, and depletion of either PKA subunit recapitulates the migratory inhibition induced by miR-200c, indicating that ZEB1 promotes tumor cell migration through a miR-200c/PKA-dependent pathway (34). Conversely, activation of the cAMP-dependent pathway can either promote or inhibit EMT. For example, PKA potentiates hypoxia-induced EMT by phosphorylating SNAIL1 (35), whereas treatment with forskolin or cholera toxin induces mesenchymal-to-epithelial transition and decreases the tumor-initiating activity of breast cancer cells through the histone demethylase PHF2, a PKA substrate (36). Similarly, cAMP blunts EMT in fibrosis models through the cAMP effector proteins PKA and EPAC (exchange protein activated by cAMP) (37). Thus, cAMP can either repress or promote EMT through pathways that are connected at multiple levels and interact bidirectionally.

These findings provide a rationale for the development of novel therapeutic strategies to inhibit AC9 in lung cancer. Isoform-selective $\mathrm{AC}$ antagonists that target the active site or allosteric sites (e.g., the diterpene site) are under development (38). Given that certain AC isoforms have metastasis suppressor activities in breast cancer (36), future preclinical and clinical trials should take into consideration the isoform-selectivity of such inhibitors. 
A
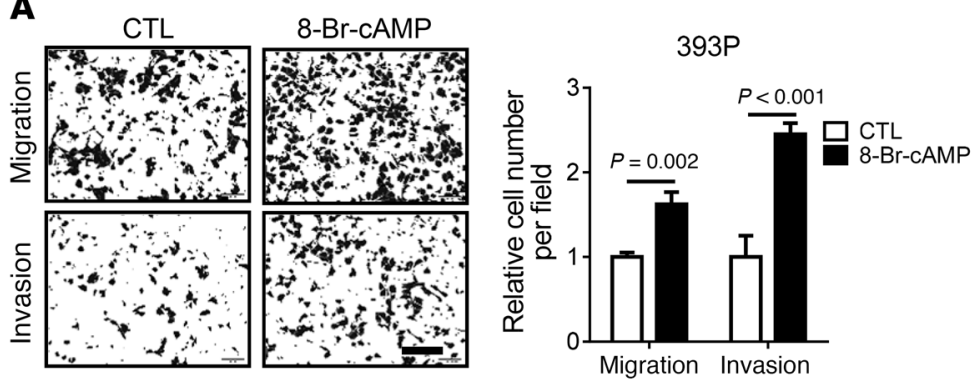

B

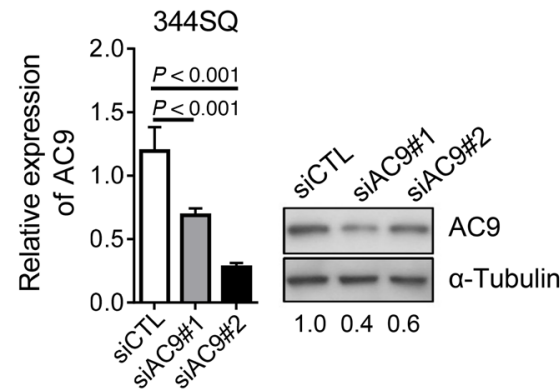

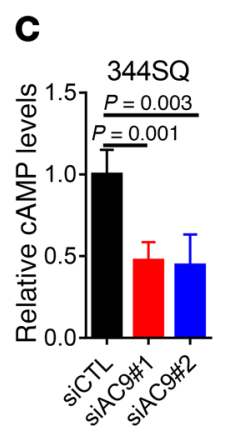

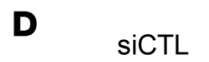

siAC9\#1 SiAC9\#2

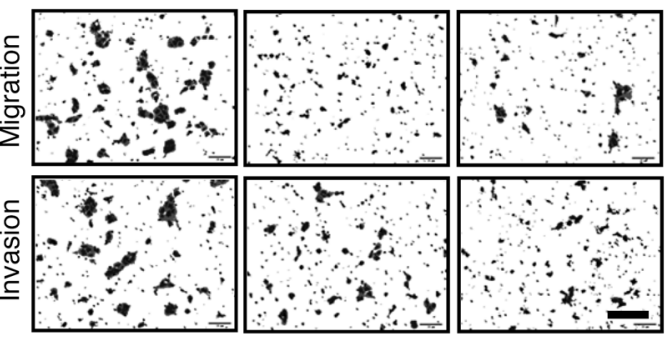

344SQ

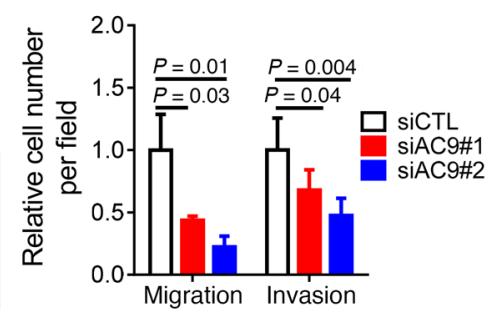

E

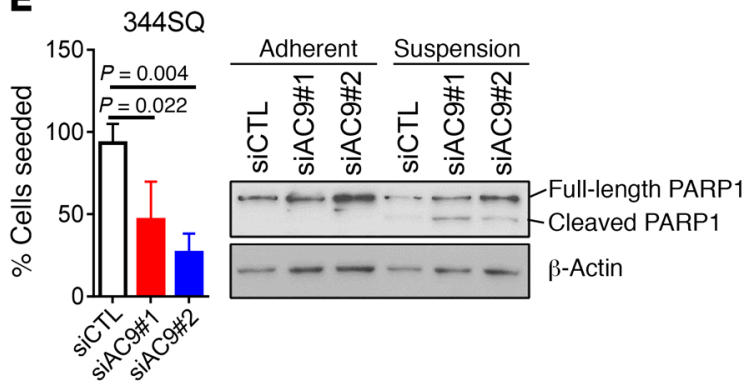

G
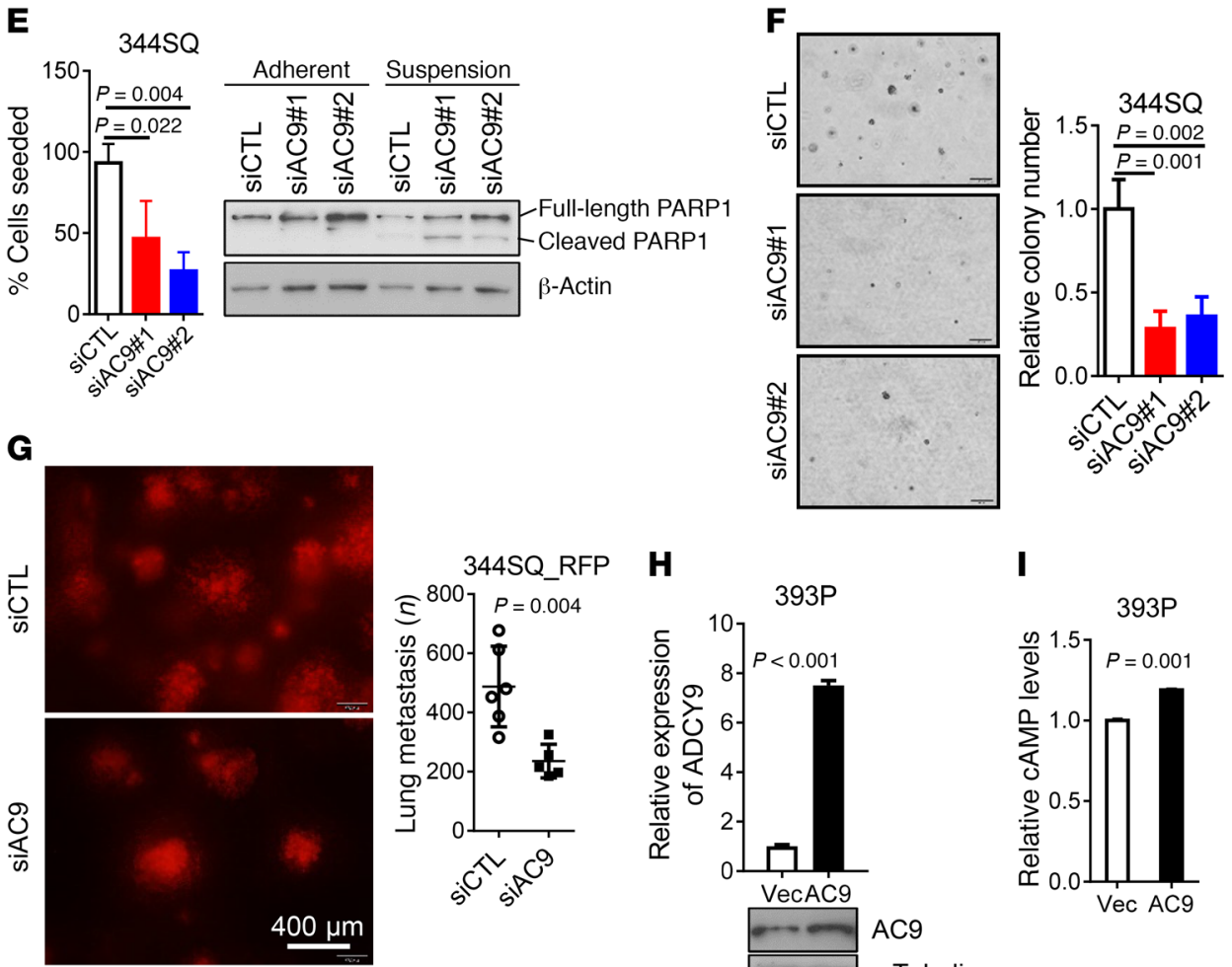

J

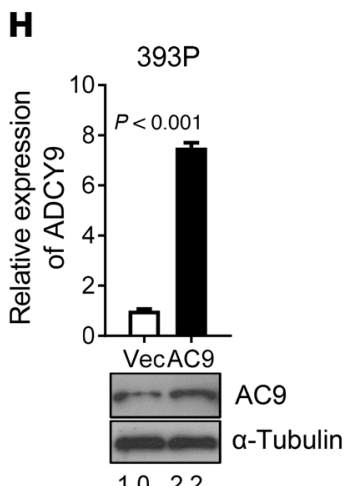

I

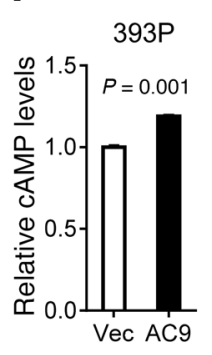

AC9

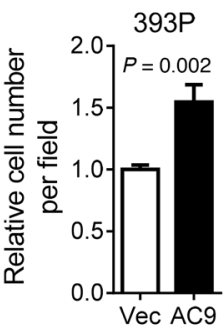


Figure 6. AC9 is essential for EMT-driven metastasis. (A) Boyden chamber assays of 393P cells treated with 8-bromo-cAMP or vehicle (CTL). Migratory cells were photographed (images) and counted (bar graph). Scale bar: 200 $\mu \mathrm{m}$. (B) qPCR (bar graph) and Western analyses (gels) of AC9 expression in $344 \mathrm{SQ}$ cells stably transfected with scrambled (siCTL) or 1 of 2 AC9 siRNAs. (C) CAMP levels in $344 \mathrm{SQ}$ cells transfected with siCTL or siAC9. (D) Boyden chamber assays of $344 \mathrm{SQ}$ cells transfected with siAC9 or siCTL. Scale bar: $200 \mu \mathrm{m}$. (E) Bar graph: numbers of viable 344SQ transfectants seeded on low-adhesion plates and counted 24 hours later. $n=4$ samples per condition. Gels: Western analysis to detect cleaved PARP as a marker of apoptosis in transfectants seeded on adhesive or low-adhesion (suspension) plates. $\beta$-Actin included as loading control. (F) Soft agar colony formation by $344 \mathrm{SQ}$ cells transfected with siAC9 or siCTL. Colonies were photographed (images) and counted (bar graph). Scale bar: $100 \mu \mathrm{m}$. (C) Fluorescence microscopic images of lung metastases in mice injected with RFP-labeled 344 SQ cells transfected with siAC9 or siCTL. Scatter plot: numbers of lung metastasis per mouse (dots). (H) qPCR and Western analyses of AC9 expression in 393P_AC9 cells (AC9) and 393P_vector cells (Vec). (I) cAMP levels in 393P_AC9 cells and 393P_vector cells. (J) Boyden chamber assays of 393P_AC9 cells and 393P_vector cells. Scale bar: $200 \mu \mathrm{m}$. Values are mean \pm SD. $n=3$, unless otherwise indicated. $P$ values, 2-tailed Student's $t$ test and Dunnett's test for 2-group and more-than-2-group comparisons, respectively. Results were replicated ( $n \geq 2$ experiments).

\section{Methods}

Antibodies, plasmids, and oligomers. Antibodies against $\alpha_{1}$ integrin (Santa Cruz Biotechnology, sc-271034), $\beta$-actin (Cell Signaling Technology, 4970), RNA polymerase II (Millipore, 05-623, clone CTD4H8), BCL2 (Cell Signaling Technology, 3498), MYC-tag (Cell Signaling Technology, 2272), PARP-1 (Cell Signaling Technology, 9542), ARNT (Cell Signaling Technology, 3414), EGFP (Sigma-Aldrich, G1544), ADCY9 (Abcam, ab191423), and AGO2 (Abcam, ab57113) were used. The following gene-specific shRNAs, siRNAs, and miR mimics were purchased from Sigma-Aldrich: mouse shITGA1 nos. 1-5 (TRCNO000254183, TRCNO000265481, TRCN0000254182, TRCNO000254180, and TRCNOOO0254181); mouse shTET2 nos. 1-3 (TRCNO000250895, TRCN0000250892, and TRCN0000217530); mouse siITGA1 nos. 1 and 2 (SASI Mm01_00102775 and SASI_Mm02_00288356); human siITGA1 nos. 1 and 2 (SASI_Hs01_00067020 and SASI_Hs01_00067021); mouse siDNMT1 (SASI_Mm01_00024007); mouse siDNMT3B (SASI_Mm02_00286413); mouse siADCY9 (SASI Mm01_00074440 and SASI_Mm01_00074441); mouse siADCY3 (SASI_Mm01_00139448 and SASI_Mm01_00139449); mouse siADCY7 (SASI_Mm02_00311533 and SASI_Mm02_00311534); and miR mimics (HMIO237 and HMIO270) and inhibitors (HSTUD0016, HSTUD0270, and HSTUD0387). The TET2 expression vector pcDNA3-Tet2 was a gift from Yi Zhang (Boston Children's Hospital, Boston, Massachusetts, USA) (Addgene plasmid 60939) (39). pcDNA3/ Myc-DNMT1 (Addgene plasmid 36939) and pcDNA3/Myc-DNMT3B1 (Addgene plasmid 35522) were gifts from Arthur Riggs (City of Hope, Duarte, California, USA) (40). pMS2-GFP (Addgene plasmid 27121) (41) and pSL-MS2-12X (Addgene plasmid 27119) (42) were gifts from Robert Singer (Albert Einstein College of Medicine, Bronx, New York, USA).pSL-MS2-12X was double-digested with EcoRI and XhoI, and the MS2-12X fragment was subcloned downstream of the ITGA1 3'-UTR in the pcDNA3.1 vector. The ITGA1 and ADCY9 coding sequences and pre-miR-181b were amplified from a cDNA isolated from 344SQ cells and subcloned into the pLVX-puro vector (Clontech). ITGA1 promoter fragments were amplified from the genomic DNA of 344SQ cells and subcloned into the pGL3-Basic vector (Promega). Transcription binding site mutations were created with a PCR-based site-directed mutagenesis method. The PCR primers are listed in Supplemental Table 3.

RNA-Seq. Total RNA samples from 393P_ZEB1 cells and 393P_vector cells (triplicate samples of each) were subjected to RNA sequencing by MD Anderson SOLiD Sequencing Core with the ABI SOLiD whole transcriptome sequencing protocol (Thermo Fisher Scientific). Paired-end short reads were produced in the form of XSQ files. XSQ files were processed with LifeScope Genomic Analysis Software version 2.5.1. The reads were aligned to the mouse genome MM10 and then counted by genes and exons using the gene transfer format (GTF) file downloaded from the UCSC Genome Browser. RPKM was also calculated. Raw data are available in the NCBI's Gene Expression Omnibus database (GSE102337).

miR expression profiling. Serial sections (10-20 $\mu \mathrm{m}$ thick) of surgically resected human lung cancer specimens $(n=247)$ were obtained using a Leica cryostat and homogenized using an Omni TH homogenizer (Omni International). Clinical characteristics of the patient cohort have been reported (43). Total RNA was isolated using TRIzol Reagent (Life Technologies). miR expression profiling was conducted using Agilent Human miRNA microarray version 3 chips (Agilent Technologies) as described previously $(43,44)$.

mRNA expression profiling. Total RNA was isolated from 393P ADCY9 cells and 393P_vector cells (triplicate samples of each) by the RNeasy Mini Kit (Qiagen). RNA quality and quantity were evaluated on a NanoDrop spectrophotometer (Thermo Fisher Scientific). RNA fluorescent labeling reaction and hybridization were performed (Affymetrix Clariom S WT Plus) according to the manufacturer's instructions and analyzed as we have described (18). Raw data are available in Gene Expression Omnibus (GSE102168).

Cell culture and functional assays. Murine lung cancer cell lines (713P, 307P, 344LN, 393LN, 393P, 412P, 344SQ, 344P, 531LN1, 531LN2, 531P1, and 531P2) were derived from KP mice as described previously (18). Human lung cancer cells (HCC827 and H1299) were purchased from the American Type Culture Collection and cultured in RPMI 1640 (Corning) supplemented with 10\% FBS (Gibco). 393P_vector cells, 393P_ZEB1 cells, 307P_vector cells, 307P_ZEB1 cells, HCC827_vector cells, and HCC827_ZEB1 cells were described previously $(11,12,45)$. Cells were transfected using jetPRIME Versatile DNA/siRNA transfection reagent (Polyplus). Stable cell transfectants were selected with puromycin (InvivoGene) or G418 (Corning). When indicated, $2 \mu \mathrm{M}$ 5-aza-2'-deoxycytidine (SigmaAldrich), $1 \mu \mathrm{g} / \mathrm{ml}$ doxycycline (Sigma-Aldrich), $1 \mu \mathrm{g} / \mathrm{ml}$ forskolin (Sigma-Aldrich), or $200 \mu \mathrm{M}$ 8-bromo-cAMP (Sigma-Aldrich) was added to the culture medium. Two thousand cells were plated in 96-well plates, and the WST-1 reagent (Roche) was used according to the manufacturer's instructions to measure cellular proliferation. For anoikis assays, cells were plated in low-adhesion plates (Corning) and counted each day for 2 days. For soft agarose colony formation assays, $5 \times 10^{4}$ cells suspended in $0.4 \%$ agarose were seeded into 6 -well plates layered with $0.8 \%$ agarose, and colonies were stained with $0.005 \%$ crystal violet after $2-3$ weeks. For migration and invasion assays, $2 \times 10^{4}$ cells were cultured in the upper wells of Transwell or Matrigel chambers (BD Biosciences), respectively, and allowed to migrate toward 10\% FBS in the bottom wells. After 8-10 hours of incubation, migrating or invading cells were stained with $0.1 \%$ crystal violet, photographed, and counted. For the cAMP 


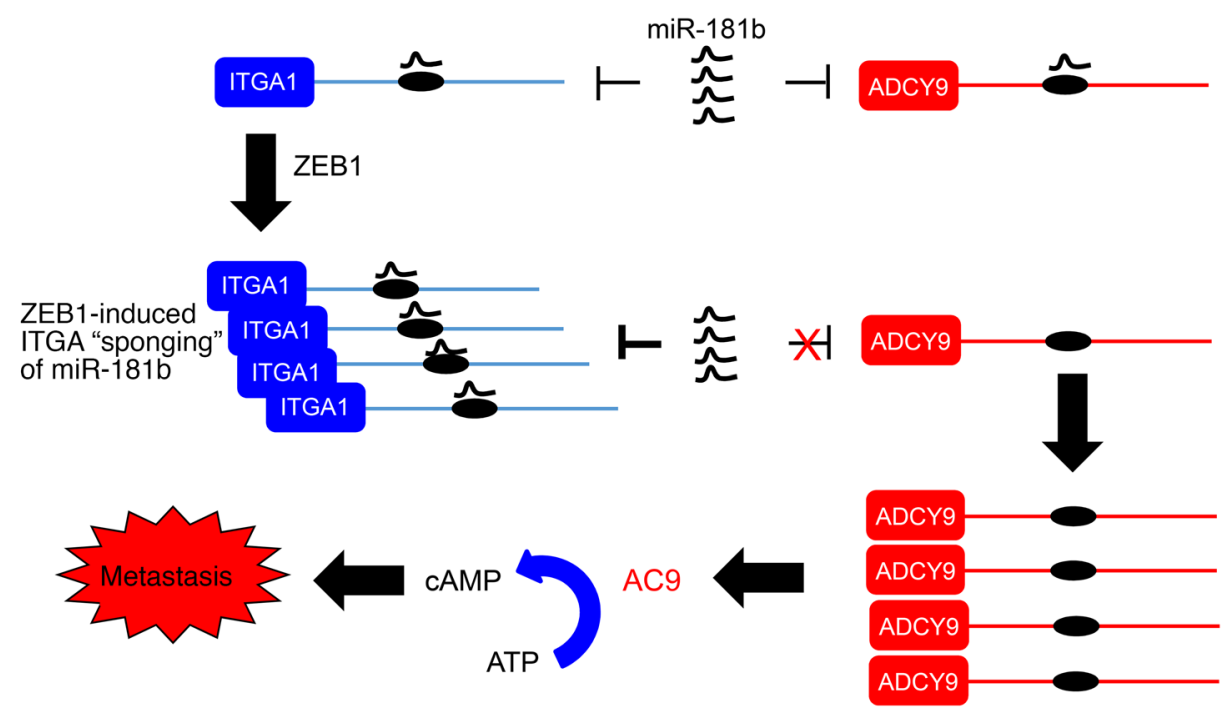

Figure 7. Working model of an EMT-driven ceRNA network. Before undergoing EMT, tumor cells have relatively low levels of the miR-181b targets ITCA1 and ADCY9, which encode $\alpha$ integrin and adenylyl cyclase 9 (AC9), respectively. The EMT activator ZEB1 upregulates ITCA1 mRNA, which sponges miR-181b and thereby relieves repression of ADCY9. High AC9 levels increase CAMP production and promote metastasis.

assay, 10,000 cells were seeded into 96-well plates, and intracellular cAMP levels were detected using cAMP-Glo Max Assay (Promega) according to the manufacturer's instructions.

qPCR analysis. Total RNA was isolated from cells using TRIzol and subjected to reverse transcription using the qScript cDNA superMix (Quanta Biosciences). mRNA levels were determined using SYBR Green Real-Time PCR Master Mixes (Thermo Fisher Scientific) and normalized on the basis of ribosomal protein L32 (Rpl32) mRNA. miR levels were quantified with stem-loop qPCR assays and normalized to U6 small nuclear RNA levels. The primer sequences for qPCR are listed in Supplemental Table 3.

Luciferase reporter assays. For the promoter assays, cells were seeded onto 48 -well plates $\left(5 \times 10^{4}\right.$ cells per well $)$ and transiently transfected 24 hours later with $200 \mathrm{ng}$ of luciferase reporter plasmids, 50 ng of pRL-TK control vector, and $250 \mathrm{ng}$ of ZEB1 expression vector or empty vector. For the 3'-UTR assays, 3'-UTR reporters (10 $\mathrm{ng})$, pGL3 control (50 ng), and miR mimics (10 nM) or miR expression vectors $(250 \mathrm{ng})$ were cotransfected into cells. After 24 hours, luciferase activity was measured with the Dual-Luciferase Reporter Assay System (Promega).

In vitro transcription and transcript copy-number analysis. Fragments of ADCY9 and ITGA1 were amplified using primers with T7 promoters and transcribed in vitro using the HiScribe T7 High Yield RNA Synthesis Kit (New England BioLabs) according to the manufacturer's instructions. RNA was subsequently purified using the RNeasy Cleanup Kit (Qiagen) according to the manufacturer's instructions. To determine the transcript copy number, we prepared RNA from 1 $\times 10^{6} 393 \mathrm{P}$ and 344 SQ cells using the RNeasy Cleanup Kit. Total cell RNAs and in vitro-transcribed RNAs were reverse-transcribed using M-MLV Reverse Transcriptase (Promega). qPCR was performed for ITGA1 and miR-181b. Threshold cycle values were compared with a 2 -fold dilution series of either in vitro-transcribed ITGA1 or miR-181b mimics (Sigma-Aldrich). Moles of transcript and copies of transcript per cell were then calculated using standard stoichiometric methods.

Chromatin immunoprecipitation. As described previously (11), cell lysates were subjected to cross-linking followed by sonication with a Cole-Parmer GEX-130 ultrasonic processor using 50\% power (pulse on for 10 seconds, pulse off for 10 seconds; 20 cycles) and immuno- precipitation with anti-RNA polymerase II, anti-ARNT, or anti-rabbit IgG antibodies (Santa Cruz Biotechnology). DNA was eluted and purified with the MinElute Reaction Cleanup Kit (Qiagen) and subjected to quantitative PCR. PCR primers are listed in Supplemental Table 3.

RNA immunoprecipitation. For the MS2-based RNA immunoprecipitation (RIP) assay, 393P cells were cotransfected with pcDNA3.1-MS2, pcDNA3.1-ITGA1 3'-UTR-WT-MS2, or pcDNA3.1ITGA1 3'-UTR-MT-MS2 and with pMS2-GFP vectors. Cells were harvested in ice-cold PBS and subjected to RIP experiments using an anti-GFP antibody or control anti-IgG antibody using the Magna RIP RNA-Binding Protein Immunoprecipitation Kit (Millipore, 17-700) according to the manufacturer's instructions. For the AGO2 RIP assay, the 344SQ cells transfected with ITGA1 siRNA or control siRNA or 393P cells transfected with WT or mutated ITGA1 3'-UTR or empty vector were harvested and subjected to RIP assay using an anti-AGO2 antibody.

Bisulfite sequencing. Genomic DNA was extracted from 393P, 344SQ, 393P_vector, and 393P_ZEB1 cells with the QIAamp DNA Mini Kit (Qiagen) and treated with bisulfite using the EpiTect Bisulfite Kit (Qiagen). The ITGA1 promoter sequence was amplified from bisulfiteconverted genomic DNA, and the PCR products were subcloned into $\mathrm{T}$ vector (Promega). Single clones were selected and subjected to direct Sanger sequencing.

Animal husbandry. All mouse studies were approved by the Institutional Animal Care and Use Committee at The University of Texas MD Anderson Cancer Center. Immunocompetent 129/Sv mice syngeneic to the KP cells were bred in house and were injected subcutaneously in the right flank with tumor cells ( $n=8-10$ mice per group) and necropsied after 5 weeks or injected intravenously with tumor cells $(n=5-8$ mice per group) and necropsied after 7-10 days. Primary tumors were weighed, and lung metastases on the pleural surfaces were counted. Mice received standard care and were euthanized according to the standards set forth by the Institutional Animal Care and Use Committee.

Statistics. The results are representative of replicated experiments and are the means \pm SD from triplicate samples or randomly chosen cells within a field unless otherwise indicated. Statistical evaluations were carried out with Prism 6 (GraphPad Software Inc.). Unpaired 2-tailed Student's $t$ tests were used to compare the means 
of 2 groups. ANOVA with Dunnett's tests was used to compare multiple treatments with a control.

The database of biological targets of miRs downloaded from TargetScan (www.targetscan.org) was used to predict biological targets of miRs (46). For miR target enrichment analysis, we performed data bioinformatics analyses using the R and Bioconductor package (https:// www.r-project.org/; https://www.bioconductor.org/), a publicly available statistical computing tool that is widely used for high-throughput "omics" data analysis. To determine whether ZEB1-regulated genes are enriched in specific miR targets, we first used an $\mathrm{R}$ package, DESeq2, to identify genes that were differentially expressed between groups (393P_vector versus 393P_ZEB1). The Benjamini-Hochberg method (47) was then applied to adjust for multiple hypothesis testing and to estimate false discovery rate. Genes with a false discovery rate ( $q$ value) less than or equal to 0.001 were considered to be differentially expressed between 393P_vector and 393P_ZEB1. We then used the hypergeometric test to test for enrichment of differentially expressed genes in predicted miR targets that were identified using a prediction algorithm (www.targetscan.org).

To determine whether ZEB1, ITGA1, and ADCY9 levels were correlated in human cancers, we used RNA-Seq data from The Cancer Genome Atlas that included acute myeloid leukemia (LAML, $n=173$ ); adrenocortical carcinoma (ACC, $n=79$ ); bladder urothelial carcinoma (BLCA, $n=408$ ); brain lower-grade glioma (LGG, $n=516$ ); breast invasive carcinoma (BRCA, $n=1095)$; cervical squamous cell carcinoma and endocervical adenocarcinoma (CESC, $n=304$ ); cholangiocarcinoma (CHOL, $n=36$ ); colorectal adenocarcinoma (CRC, combining COAD and READ projects, $n=623$ ); esophageal carcinoma (ESCA, $n=184$ ); glioblastoma multiforme (GBM, $n=161$ ); head and neck squamous cell carcinoma (HNSC, $n=520$ ); kidney chromophobe (KICH, $n=66$ ); kidney renal clear cell carcinoma (KIRC, $n=533$ ); kidney renal papillary cell carcinoma (KIRP, $n=290$ ); liver hepatocellular carcinoma (LIHC, $n=371$ ); lung adenocarcinoma (LUAD, $n=515$ ); lung squamous cell carcinoma (LUSC, $n=501$ ); lymphoid neoplasm diffuse large B cell lymphoma (DLBC, $n=48$ ); mesothelioma (MESO, $n=87$ ); ovarian serous cystadenocarcinoma (OV, $n=262)$; pancreatic adenocarcinoma (PAAD, $n=178$ ); pheochromocytoma and paraganglioma (PCPG, $n=179$ ); prostate adenocarcinoma (PRAD, $n=497$ ); sarcoma (SARC, $n=259$ ); skin cutaneous melanoma (SKCM, $n=469)$; stomach adenocarcinoma (STAD, $n=$ 415); testicular germ cell tumors (TGCT, $n=150$ ); thymoma (THYM, $n=$
120); thyroid carcinoma (THCA, $n=503$ ); uterine carcinosarcoma (UCS, $n=57$ ); and uterine corpus endometrial carcinoma (UCEC, $n=545)$. $P$ less than 0.05 was considered statistically significant.

To analyze a compendium of human lung adenocarcinoma cohorts (48) for the presence or absence of the ADCY9-derived expression signature, we used a $t$ score described elsewhere (29).

\section{Author contributions}

JNK and XT conceived and designed the study. XT, PB, and Xiuping Liu developed the methodology. JMK, XT, Xin Liu, PB, MS, CB, JF, and IIW acquired data (provided animals, acquired and managed patients, provided facilities, etc.). XT, PB, CJC, LD, XZ, JY, PW, and KLS analyzed and interpreted the data (e.g., statistical analysis, biostatistics, computational analysis). JMK and XT wrote, reviewed, and/or revised the manuscript. JMK, XT, PB, Xin Liu, DLG, CJC, AJ, MS, CB, JF, LD, JW, Xiuping Liu, and CGL provided administrative, technical, or material support (i.e., reporting or organizing data, constructing databases). JMK supervised the study.

\section{Acknowledgments}

We thank Fengju Chen for technical assistance. This work was supported by the NIH through R01 CA181184 (to J.M. Kurie), P30 CA125123 (to C.J. Creighton), NIH Lung Cancer SPORE grant P50 CA70907 (to J.M. Kurie and I.I. Wistuba), K08 CA151661 (to D.L. Gibbons), Department of Defense PROSPECT grant W81XWH-07-1-0306 (to I.I. Wistuba), and Cancer Prevention Research Institute of Texas Multi-investigator Research Award RP120713 (to J.M. Kurie, C.J. Creighton, I.I. Wistuba, and D.L. Gibbons). J.M. Kurie holds the Elza A. and Ina S. Freeman Endowed Professorship in Lung Cancer. D.L. Gibbons is an R. Lee Clark Fellow of The University of Texas MD Anderson Cancer Center supported by the Jeanne F. Shelby Scholarship Fund. We acknowledge the Department of Scientific Publications at The University of Texas MD Anderson Cancer Center for helpful assistance in manuscript preparation.

Address correspondence to: Jonathan M. Kurie, The University of Texas MD Anderson Cancer Center, Box 432, Department of Thoracic/Head and Neck Medical Oncology, 1515 Holcombe Boulevard, Houston, Texas 77030, USA. Phone: 731.745.6747; Email: jkurie@mdanderson.org.
1. Loberg RD, Bradley DA, Tomlins SA, Chinnaiyan AM, Pienta KJ. The lethal phenotype of cancer: the molecular basis of death due to malignancy. CA Cancer J Clin. 2007;57(4):225-241.

2. Brabletz T. To differentiate or not - routes towards metastasis. Nat Rev Cancer. 2012;12(6):425-436

3. Brabletz T. EMT and MET in metastasis: where are the cancer stem cells? Cancer Cell. 2012;22(6):699-701.

4. Wellner U, et al. The EMT-activator ZEB1 promotes tumorigenicity by repressing stemness-inhibiting microRNAs. Nat Cell Biol. 2009;11(12):1487-1495.

5. Chen L, et al. Metastasis is regulated via microRNA-200/ZEB1 axis control of tumour cell PD-L1 expression and intratumoral immunosuppression. Nat Commun. 2014;5:5241.

6. Fischer KR, et al. Epithelial-to-mesenchymal transition is not required for lung metastasis but contributes to chemoresistance. Nature. 2015;527(7579):472-476.

7. Kalluri R, Weinberg RA. The basics of epithelialmesenchymal transition. J Clin Invest. 2009;119(6):1420-1428.

8. Krebs AM, et al. The EMT-activator Zeb1 is a key factor for cell plasticity and promotes metastasis in pancreatic cancer. Nat Cell Biol. 2017;19(5):518-529.

9. Zheng X, et al. Epithelial-to-mesenchymal transition is dispensable for metastasis but induces chemoresistance in pancreatic cancer. Nature. 2015;527(7579):525-530.

10. Browne G, Sayan AE, Tulchinsky E. ZEB proteins link cell motility with cell cycle control and cell survival in cancer. Cell Cycle. 2010;9(5):886-891.

11. Ahn YH, et al. ZEB1 drives prometastatic actin cytoskeletal remodeling by downregulating miR-34a expression. JClin Invest. 2012;122(9):3170-3183.

12. Tan X, et al. Epithelial-to-mesenchymal transition drives a pro-metastatic Golgi compaction process through scaffolding protein PAQR11. J Clin Invest. 2017;127(1):117-131.

13. Poliseno L, Salmena L, Zhang J, Carver B, Haveman WJ, Pandolfi PP. A coding-independent function of gene and pseudogene mRNAs regulates tumour biology. Nature. 2010;465(7301):1033-1038.

14. Yuan JH, et al. A long noncoding RNA activated by TGF- $\beta$ promotes the invasion-metastasis cascade in hepatocellular carcinoma. Cancer Cell. 2014;25(5):666-681.

15. Hsiao KY, et al. Noncoding effects of circular RNA CCDC66 promote colon cancer growth and metastasis. Cancer Res. 2017;77(9):2339-2350.

16. Chen L, et al. circRNA_100290 plays a role in oral cancer by functioning as a sponge of the miR-29 family. Oncogene. 2017;36(32):4551-4561. 
17. Powers JT, et al. Multiple mechanisms disrupt the let-7 microRNA family in neuroblastoma. Nature. 2016;535(7611):246-251.

18. Gibbons DL, et al. Contextual extracellular cues promote tumor cell EMT and metastasis by regulating miR-200 family expression. Genes Dev. 2009;23(18):2140-2151.

19. Shi ZM, et al. MiRNA-181b suppresses IGF-1R and functions as a tumor suppressor gene in gliomas. $R N A$. 2013;19(4):552-560.

20. Wang X, et al. MiR-181b regulates cisplatin chemosensitivity and metastasis by targeting TGF $\beta R 1 /$ Smad signaling pathway in NSCLC. Sci Rep. 2015;5:17618.

21. Karreth FA, Pandolfi PP. ceRNA cross-talk in cancer: when ce-bling rivalries go awry. Cancer Discov. 2013;3(10):1113-1121.

22. Tay Y, et al. Coding-independent regulation of the tumor suppressor PTEN by competing endogenous mRNAs. Cell. 2011;147(2):344-357.

23. Gardner H. Integrin $\alpha 1 \beta 1$. Adv Exp Med Biol. 2014;819:21-39.

24. Brabletz S, Brabletz T. The ZEB/miR-200 feedback loop - a motor of cellular plasticity in development and cancer? EMBO Rep. 2010;11(9):670-677.

25. Brabletz S, et al. The ZEB1/miR-200 feedback loop controls Notch signalling in cancer cells. EMBO J. 2011;30(4):770-782.

26. Robertson KD. DNA methylation and human disease. Nat Rev Genet. 2005;6(8):597-610.

27. Zhuang LK, et al. MicroRNA-181a-mediated downregulation of AC9 protein decreases intracellular cAMP level and inhibits ATRAinduced APL cell differentiation. Cell Death Dis. 2014;5:e1161.

28. Sadana R, Dessauer CW. Physiological roles for $G$ protein-regulated adenylyl cyclase isoforms: insights from knockout and overexpression studies. Neurosignals. 2009;17(1):5-22.

29. Gibbons DL, et al. Expression signatures of metastatic capacity in a genetic mouse model of lung adenocarcinoma. PLoS One. 2009;4(4):e5401.

30. Tay Y, Rinn J, Pandolfi PP. The multilayered complexity of ceRNA crosstalk and competition. Nature. 2014;505(7483):344-352.

31. Wang J, et al. CREB up-regulates long non-coding RNA, HULC expression through interaction with microRNA-372 in liver cancer. Nucleic Acids Res. 2010;38(16):5366-5383.

32. Karreth FA, et al. The BRAF pseudogene functions as a competitive endogenous RNA and induces lymphoma in vivo. Cell. 2015;161(2):319-332.

33. Rutnam ZJ, Yang BB. The non-coding 3' UTR of CD44 induces metastasis by regulating extracellular matrix functions. JCell Sci. 2012;125(pt 8): 2075-2085.

34. Sigloch FC, Burk UC, Biniossek ML, Brabletz T, Schilling O. miR-200c dampens cancer cell migration via regulation of protein kinase A subunits. Oncotarget. 2015;6(27):23874-23889.

35. MacPherson MR, et al. Phosphorylation of serine 11 and serine 92 as new positive regulators of human Snail1 function: potential involvement of casein kinase-2 and the CAMP-activated kinase protein kinase A. Mol Biol Cell. 2010;21(2):244-253.

36. Pattabiraman DR, et al. Activation of PKA leads to mesenchymal-to-epithelial transition and loss of tumor-initiating ability. Science. 2016;351(6277):a680.

37. Insel PA, et al. cAMP and Epac in the regulation of tissue fibrosis. Br J Pharmacol. 2012;166(2):447-456.

38. Seifert R, Lushington GH, Mou TC, Gille A, Sprang SR. Inhibitors of membranous adenylyl cyclases. Trends Pharmacol Sci. 2012;33(2):64-78.

39. Wang Y, Zhang Y. Regulation of TET protein stability by calpains. Cell Rep. 2014;6(2):278-284.

40. Li H, Rauch T, Chen ZX, Szabó PE, Riggs AD, Pfeifer GP. The histone methyltransferase SETDB1 and the DNA methyltransferase DNMT3A interact directly and localize to promoters silenced in cancer cells. J Biol Chem. 2006;281(28):19489-19500.

41. Fusco D, et al. Single mRNA molecules demonstrate probabilistic movement in living mammalian cells. Curr Biol. 2003;13(2):161-167.

42. Bertrand E, Chartrand P, Schaefer M, Shenoy SM, Singer RH, Long RM. Localization of ASH1 mRNA particles in living yeast. Mol Cell. 1998;2(4):437-445.

43. Du L, et al. miR-337-3p and its targets STAT3 and RAP1A modulate taxane sensitivity in non-small cell lung cancers. PLoS One. 2012;7(6):e39167.

44. Du L, et al. miR-93-directed downregulation of DAB2 defines a novel oncogenic pathway in lung cancer. Oncogene. 2014;33(34):4307-4315.

45. Kundu ST, et al. The miR-200 family and the miR-183 96 182 cluster target Foxf2 to inhibit invasion and metastasis in lung cancers. Oncogene. 2016;35(2):173-186.

46. Lewis BP, Burge CB, Bartel DP. Conserved seed pairing, often flanked by adenosines, indicates that thousands of human genes are microRNA targets. Cell. 2005;120(1):15-20.

47. Benjamini Y, Hochberg Y. Controlling the false discovery rate: a practical and powerful approach to multiple testing. JR Stat Soc Series B Methodol. 1995;57(1):289-300.

48. Yang Y, et al. ZEB1 sensitizes lung adenocarcinoma to metastasis suppression by PI3K antagonism. J Clin Invest. 2014;124(6):2696-2708. 